GA-A15771

UC-20f

\title{
ENERGY CONFINEMENT IN DOUBLET III WITH HIGH-Z LIMITERS
}

\author{
by
}

F. B. MARCUS, SANDRA J. ADCOCK, D. R. BAKER, F. P. BLAU, N. H. BROOKS, R. P. CHASE, J. C. DeBOO,

S. EJIMA, E. S. FAIRBANKS, R. K. FISHER, C. L. HSIEH, G. L. JAHNS, M. A. MAHDAVI, R. L. MILLER,

T. OHKAWA, N. OZAKI, F. PARLANGE, T. W. PETRIE, W. W. PFEIFFER, A. M. SLEEPER, L. J. SRNKA,

R. D. STAMBAUGH, T. TAMANO, T. TAYLOR, R. E. WALTZ, and J. C. WESLEY

\section{MASTER}




\section{DISCLAIMER}

This report was prepared as an account of work sponsored by an agency of the United States Government. Neither the United States Government nor any agency Thereof, nor any of their employees, makes any warranty, express or implied, or assumes any legal liability or responsibility for the accuracy, completeness, or usefulness of any information, apparatus, product, or process disclosed, or represents that its use would not infringe privately owned rights. Reference herein to any specific commercial product, process, or service by trade name, trademark, manufacturer, or otherwise does not necessarily constitute or imply its endorsement, recommendation, or favoring by the United States Government or any agency thereof. The views and opinions of authors expressed herein do not necessarily state or reflect those of the United States Government or any agency thereof. 


\section{DISCLAIMER}

Portions of this document may be illegible in electronic image products. Images are produced from the best available original document. 
GA-A15771

UC-20

\section{DISCLAIMER \\ This book was prepared as an accounl of work sponsored by an egency of the United States Government. Neither the United States Government nor any agency thereof, nor any of their employees, makes any warranty. express or implied, or assumes any legal liability of responsibility tor the scarard. copresents that its use would not infringe privately owned rights. Reference herein to any specific commercial product, process, of service by trade name, trademark, manufacturer, or othenwise. does not necessarily constitute or imply its endorsement, recommendation, or lowaring by the Unito Siates Government of any agency thereal. The views and opinions of authors expressed herein do not \\ ENERGY CONFINEMENT IN DOUBLET III WITH HIGH-Z LIMITERS}

by

F. B. MARCUS, SANDRA J. ADCOCK, D. R. BAKER, F. P. BLAU, N. H. BROOKS, R. P. CHASE, J. C. DeBOO,

S. EJIMA, E S. FAIRBANKS, R. K. FISHER, C. L. HSIEH, G. L. JAHNS, M. A. MAHDAVI, R. L. MILLER, T. OHKAWA, N. OZAKI", F. PARLANGEt, T. W. PETRIE, W. W. PFEIFFER, A. M. SLEEPER, L. J. SRNKA**, R. D. STAMBAUGH, T. TAMANO, T. TAYLOR, R. E. WALTZ, and J. C. WESLEY

"Energy Research Laboratory, Hitachi Ltd., Hitachi, Japan. - Present address: Exxon Production Research Company, Houston, TX. tOn leave from Association Euratom CEA GRENOBLE, France.

Submitted for publication in the Proceedings of the American Physical Society Division of Plasma Physics Meeting on November 8-12, 1979, Boston, Massachusetts.

Work supported by

Department of Energy Contract DE-AT03-76ET51011

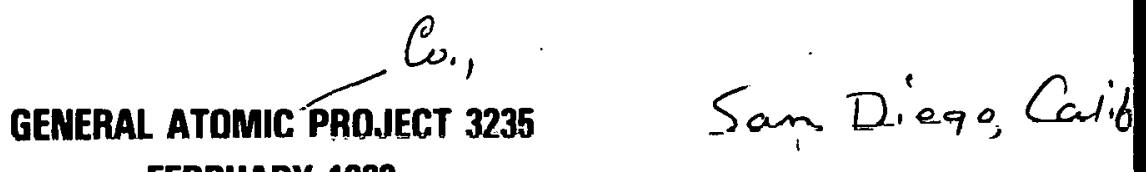
FEBRUARY 1980 
This report was prepared as an account of work sponsored by an agency of the United States Government. Neither the United States nor any agency thereof, nor any of their employees, makes any warranty, expressed or implied, or assumes any legal liability or responsibility for any third party's use or the results of such use of any information, apparatus, product or process disclosed in this report, or represents that its use by such third party would not infringe privately owned rights.

Printed in the United States of America

Available from

National Technical Information Service

U.S. Department of Commerce

5285 Port Royal Road

Springfield, Virginia 22161

Price: Printed Copy: A04; Microfiche A01 


\section{INTRODUCTION}

This report describes the experimental measurements and data analysis techniques used to evaluate the energy confinement in noncircular plasmas produced in Doublet III. The data was obtained in the first half of 1979 during plasma operation with high-z limiters. Confinement times for a variety of plasmas with near-circular $(b / a=1.1-1.3)$ and $\operatorname{doublet}(b / a=3)$ cross sections are calculated and compared. In all cases, the measurements were made during the steady-state portions of discharges with low levels of low- $Z$ and metallic impurities. The electron energy confinement is found to be equal at a given density in both the near-circular and doublet plasmas, and to increase in porportion to the plasma density, with a scaling coefficient similar to that obtained in other circular tokamaks. No deterioration in confinement due to the effects of plasma shaping is observed. The lack of a detectable improvement in the confinement of doublets relative to the near-circular plasmas is to be expected, since magnetic measurements indicate that the plasma current density is strongly peaked on axis. With such highly peaked current profiles, the interior flux surfaces of the doublet are only slightly noncircular, and the theoretically expected confinement improvement is less than the scatter and uncertainty in the experimental data.

Major aspects of the confinement measurements and analysis techniques are summarized in the following sections of this report. Machine parameters, diagnostic systems and discharge parameters relavent to the confinement measurements are given in Section 2. Magnetic analysis techniques used to determine the plasma shape are reviewed in Section 3. Scaling of the on-axis values of electron temperature, confinement time and $Z_{\text {eff }}$ with plasma density is presented in Section 4. Comparison with scaling results 
from other circular tokamaks is discussed. Numerical and analytic techniques developed for calculating the plasma energy confinement time and selfconsistent profiles of density, temperature, current, and flux in non-circular geometries are described in Sections 5 and 6 . These techniques are applied to the data and used to determine the central and global electron energy confinement time for a typical doublet plasma. Additional aspects of the confinement such as the radial dependence of the electron thermal conductivity and the estimated ion temperature are explored in Section 7 with the aid of a non-circular transport simulation code. The results of the confinement measurements are summarized and discussed in section 8 . A brief summary of the theoretically expected effects of noncircularity on plasma confinement is included for reference as Appendix I. 


\section{DOUBLET III PARAMETERS, DIAGNOSTIC SYSTEMS, AND DISCHARGE CHARACTERISTICS}

Doublet III has the following parameters:

$\begin{array}{ll}\text { major radius } & \mathrm{R}=1.43 \text { meters } \\ \text { plasma width } & 2 \mathrm{a}=0.9 \text { meters } \\ \text { plasma height } & 2 \mathrm{~b}=2.90 \text { meters } \\ \text { toroidal field } & \mathrm{B}_{\mathrm{T}} \leq 26 \mathrm{kilogauss} \\ \text { plasma current } & \mathrm{I}_{\mathrm{p}} \leq 2.2 \mathrm{MA}\end{array}$

The Inconel 625 vacuum vessel is surrounded by 24 field shaping coils (Fig. 1), and can accommodate a variety of plasma cross sections with a vertical elongation of up to 3.2. Feedback control of the magnetic flux linking the shaping coils with SCR power supplies allows the shape and position of the discharge to be controlled [1].

The composition of the primary limiters installed during the confinement studies was $90 \%$ tantalum - $10 \%$ tungsten, and the secondary limiters were of molybdenum [2]. The primary limiters are on the outer wall, with a front surface $\geq 4 \mathrm{~cm}$ from the vacuum wall. The secondary limiters are lucaled ar three toroidal azimuths, encircling the plasma poloidally, with the froint surface $3 \mathrm{~cm}$ from the wall. Each night, low power Taylor discharge cleaning $[3,4]$ is used to remove impurities from the vacuum vessel wall.

The magnetic diagnostics (Fig. 2) which provide the data for plasma shape control and MHD equilibrium analysis include 24 single-turn flux loops located on the side facing the plasma of each coil, 12 extended magnetic probes called partial Rogowski coils to measure the poloidal fleld, full Rogowski coils to measure the plasma current, and several 


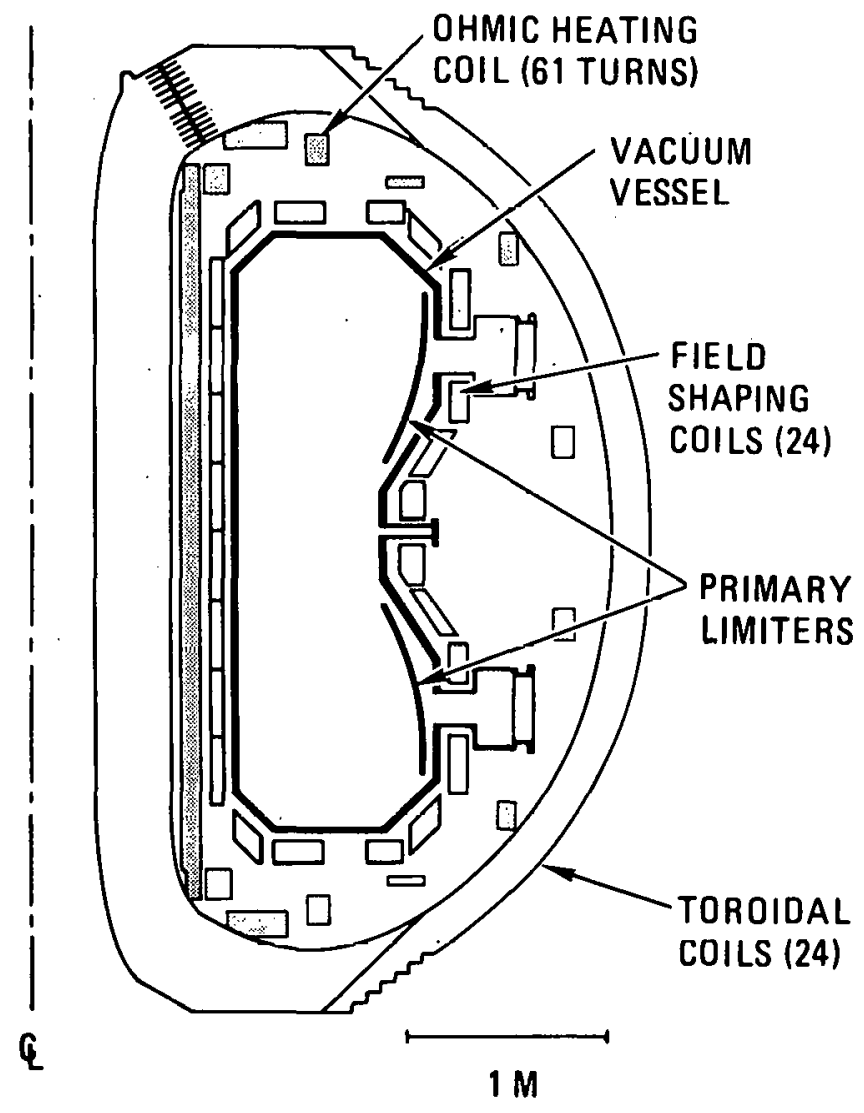

Fig. 1. Doublet III cross-section 


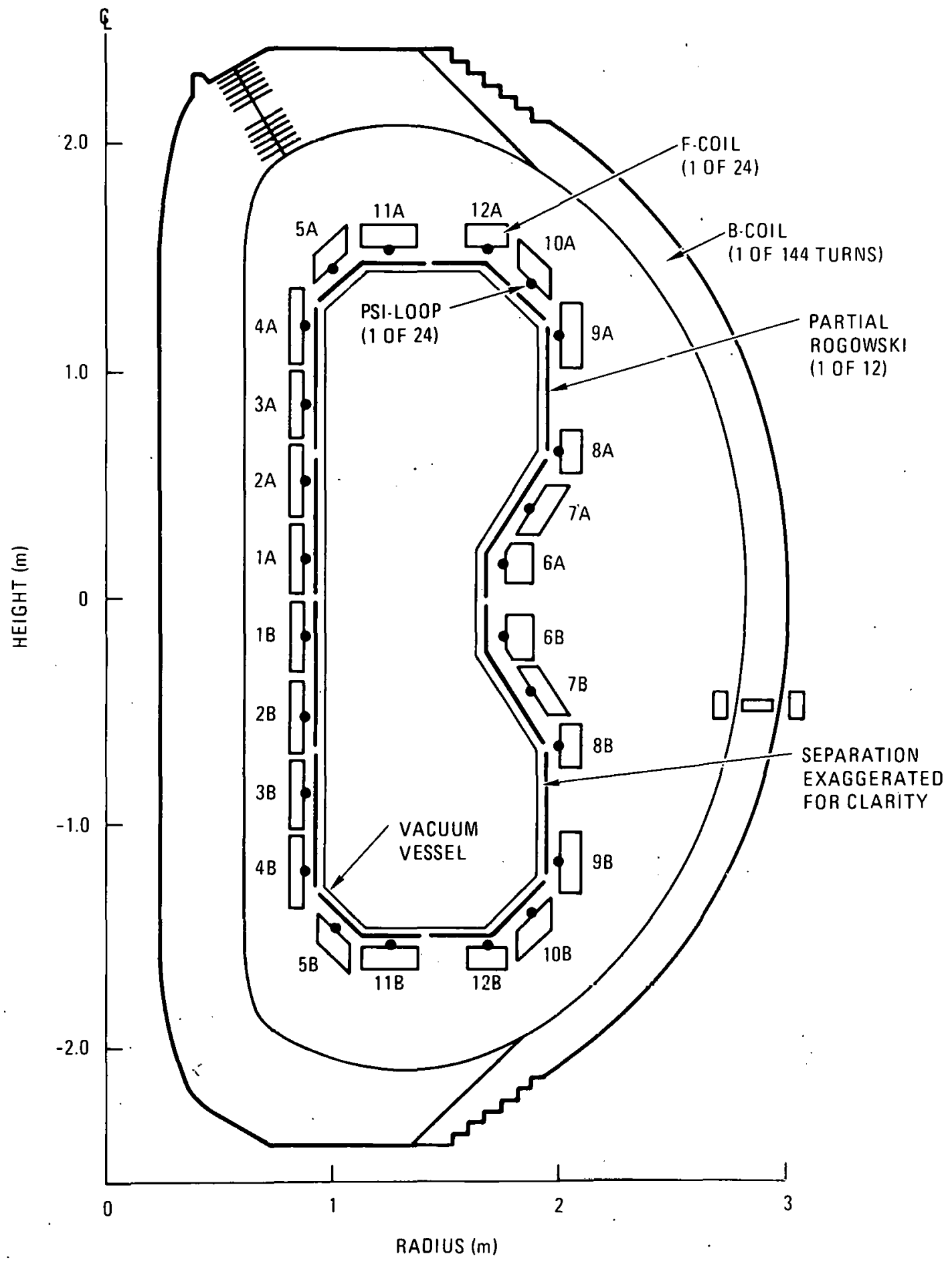

Fig. 2. Field shaping coils and magnetic diagnostics 
localized magnetic probes. The principal plasma diagnostics used for the confinement measurement are illustrated in Fig. 3. The plasma density is measured by two interferometers, a two channel horizontal-path $2 \mathrm{~mm}$ microwave system and a vertical-path 10.6 micron $\mathrm{CO}_{2}$ laser system. The electron temperature profile is measured by a far-infrared Michelson radiometer which monitors the intensity of electron cyclotron harmonic radiation emitted from the upper region of the plasma $(Z \sim 90 \mathrm{~cm})$. The electron temperature profile is computed by a Fourier analysis of the emission data collected over a $14 \mathrm{msec}$ period. The radiometer is calibrated against a single-point Thomson scattering system, which measures the electron temperature near the upper plasma center.

Confinment data has been obtained for three types of plasma discharges, iliustrated in Figs. 4 and 5. In order of increasing vertical elongation; the configurations are droplet, with two separated current channels, each with $\mathrm{b} / \mathrm{a} \cong 1.1$; ellipse or singlet, a single current channel in the upper half of the vessel with $\mathrm{b} / \mathrm{a} \cong 1.25$; and doublet, with an internal separatrix and $b / a \cong 3$.

The detailed time-dependent waveforms of a typical doublet discharge are shown in Fig. 6. Similar waveforms are obtained for droplet and elliptical discharges. The discharge lasts 0.5 seconds, and steady-state conditions occur during the last 200 milliseconds. Gas injection at a constant 40 Torr-l/sec rate results in a rising density and sufficient edge cooling to control the influx of metallic impurities. In the steady-state phase of the discharge, the loop voltage stabilizes at $1.6 \mathrm{~V} / \mathrm{turn}$, and the radial position, height and percentage of flux inside the separatrix indicated by the magnetic diagnostics all become nearly constant. The temperature profile (Fig. 7) is centrally peaked and essential time-independent. Soft $x$-ray emission from the plasma core also becomes constant. The axial value of the safety factor $\mathrm{q}(0)$ on axis is inferred to be 1.0 by observation of sawtooth oscillations in the soft $x$-ray signal in droplet plasmas. 


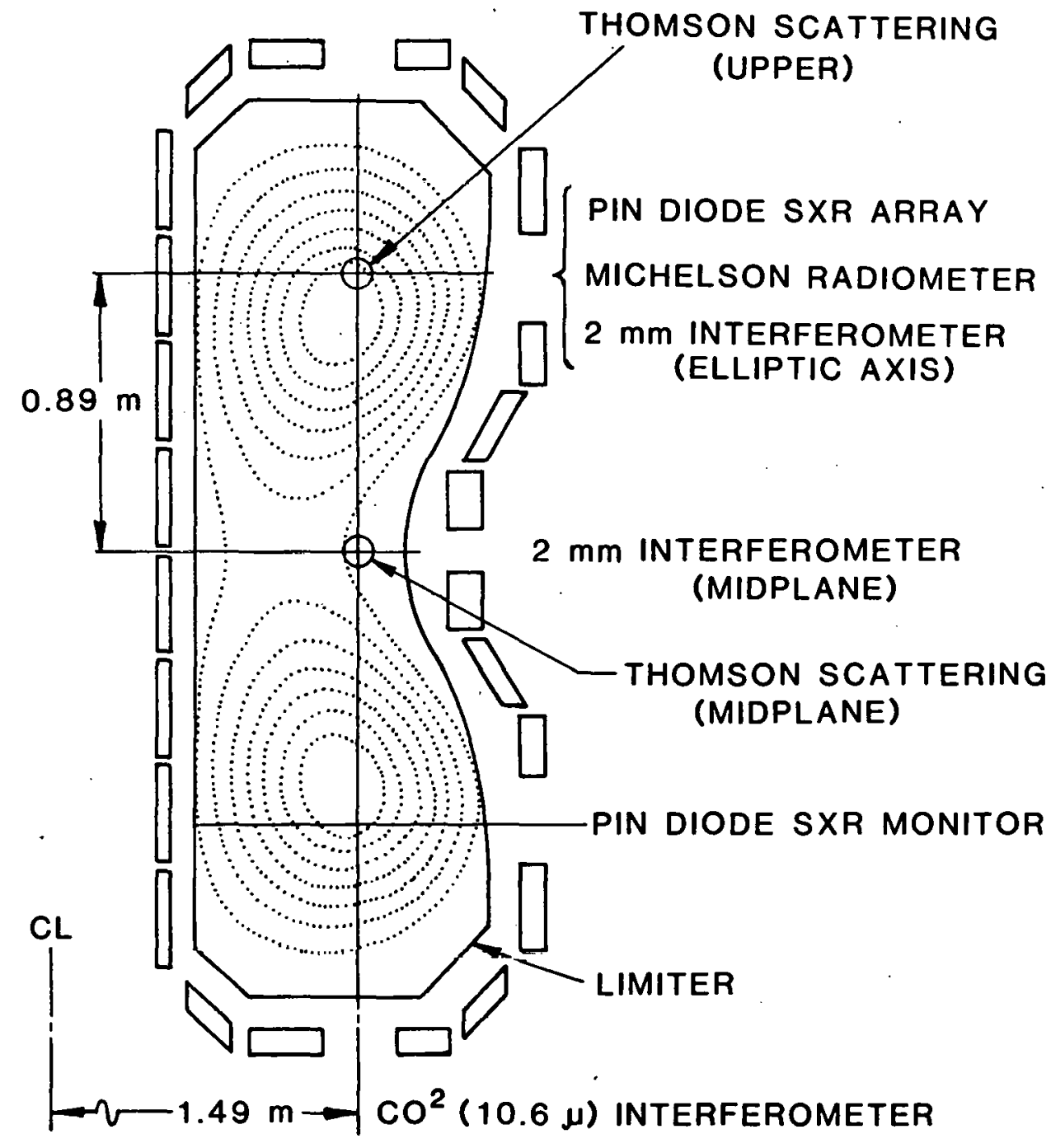

Fig. 3. Plasma dlagnustics used for energy confinement measurements 
SHOT 4103

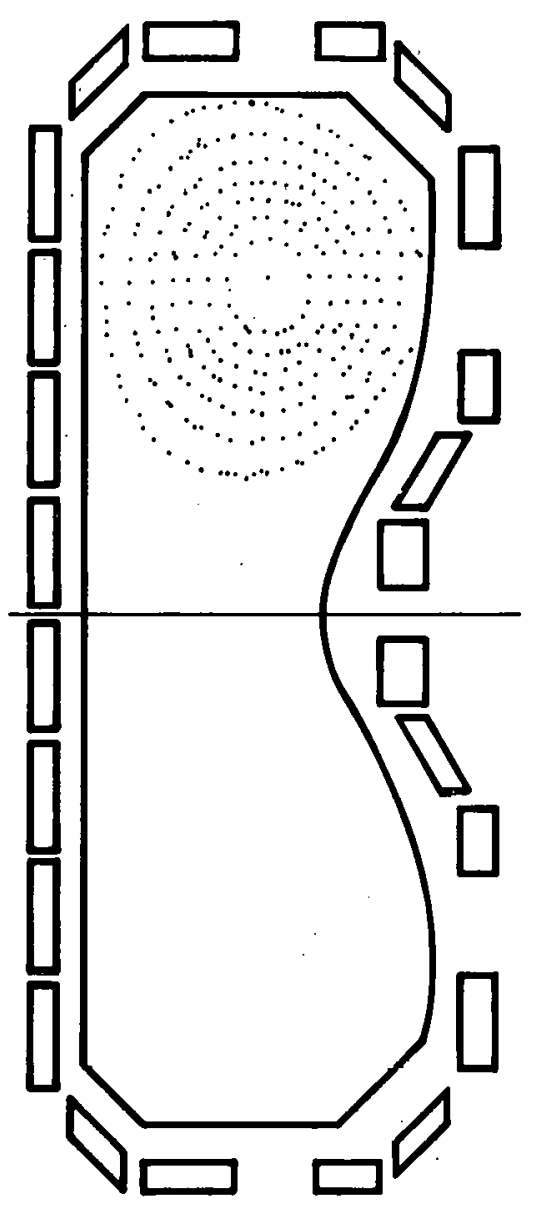

$I_{p}=400 \mathrm{kA}$
$H / W=1.25$

200 MSEC
SHOT 2618

200 MSEC

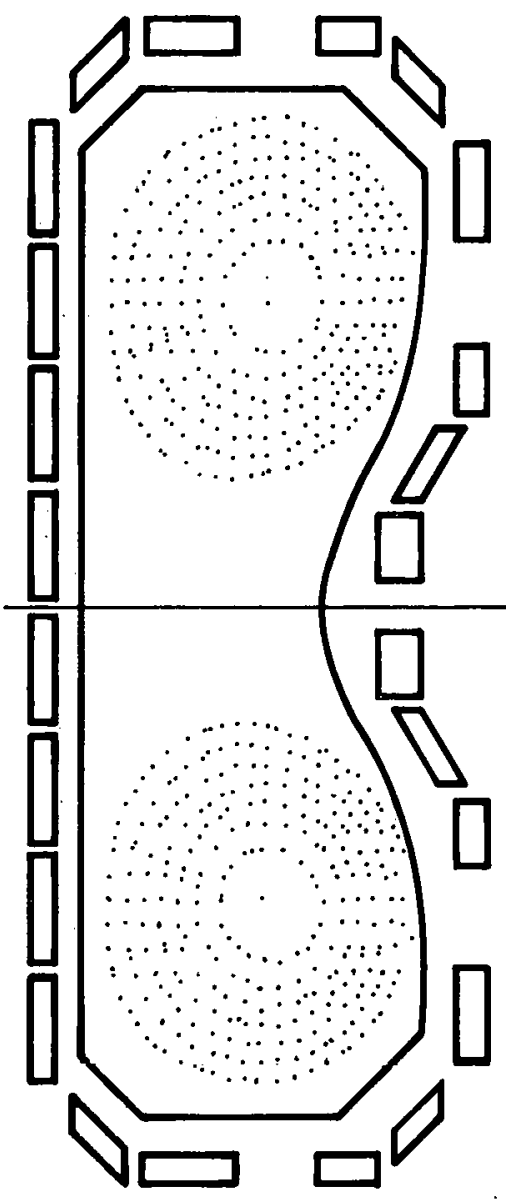

$I_{p}=650 \mathrm{kA}$
$H / W=1.1$

Fig. 4. Flux surfaces for near-circular plasmas. The MHD analysis procedure used to obtain these plots is described in Section 3 . 


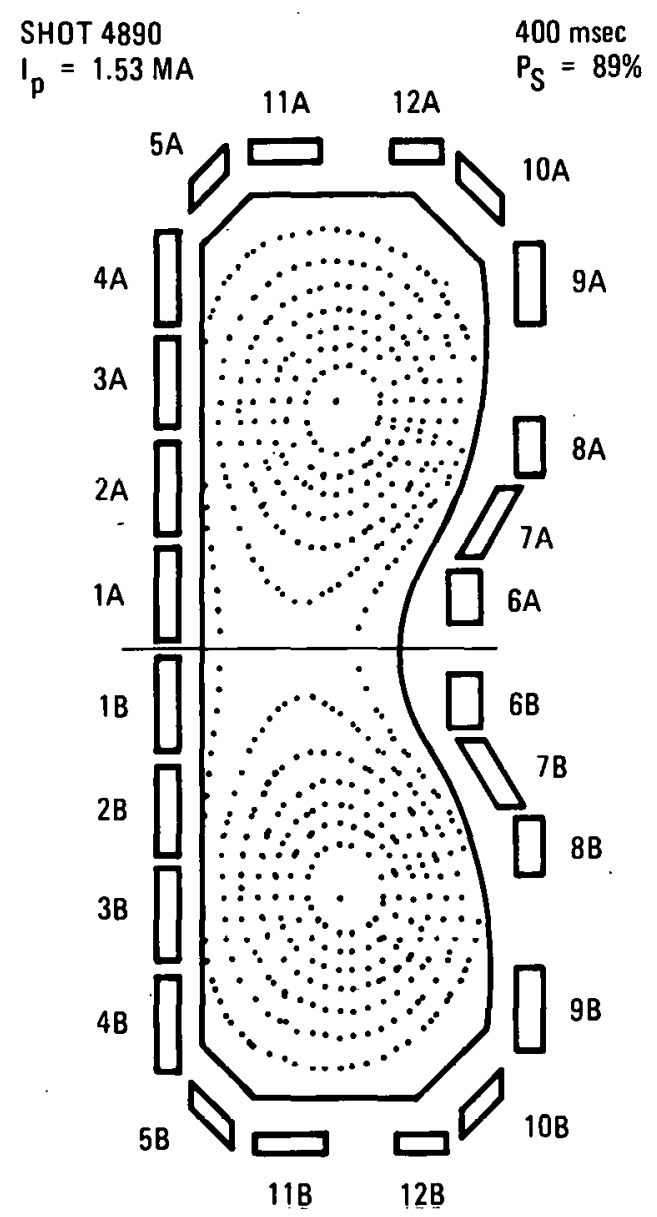

Fig. 5. Doublet flux surfacés 

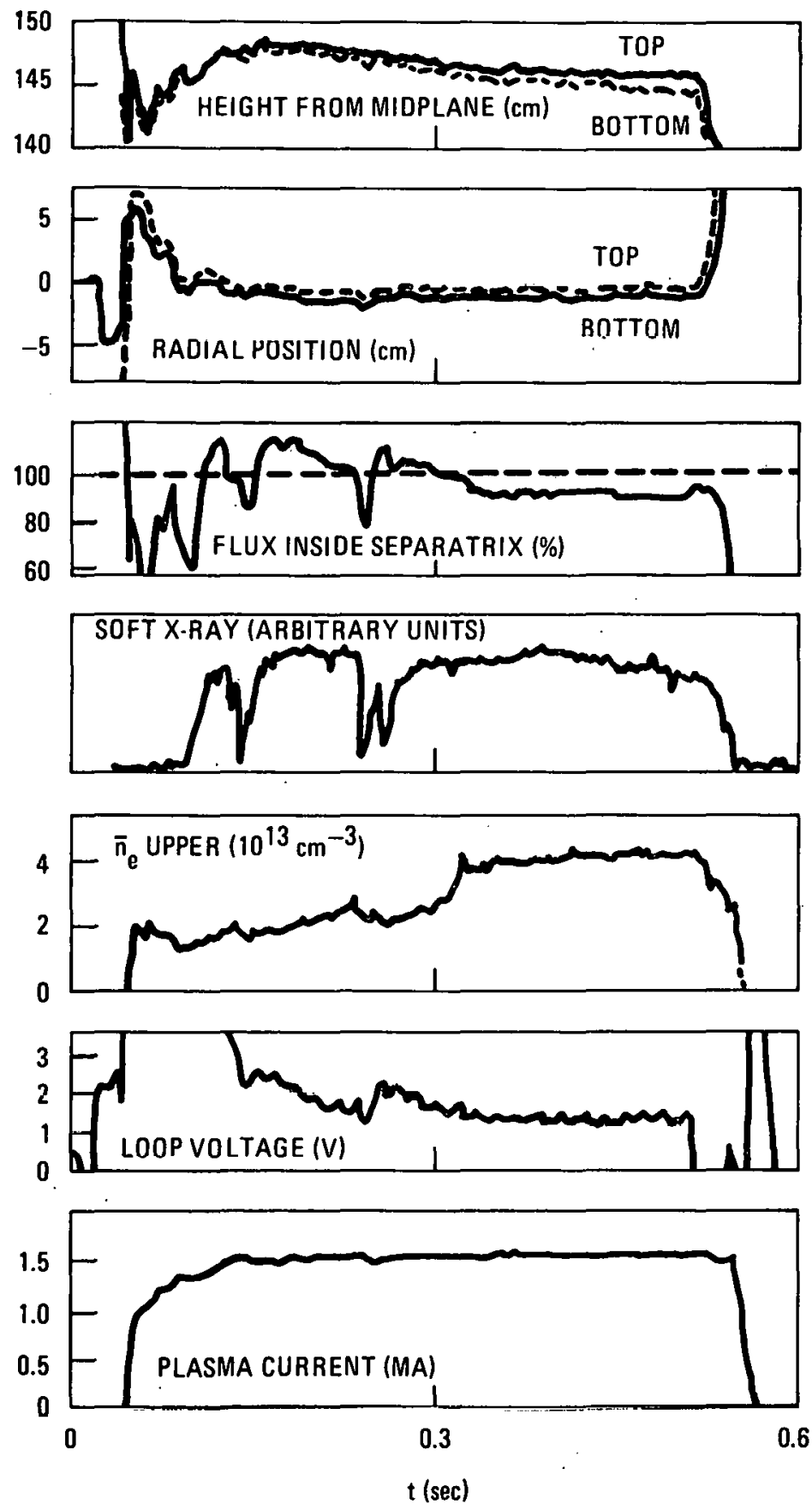

Fig. 6. Doublet discharge waveforms for shot 4890 


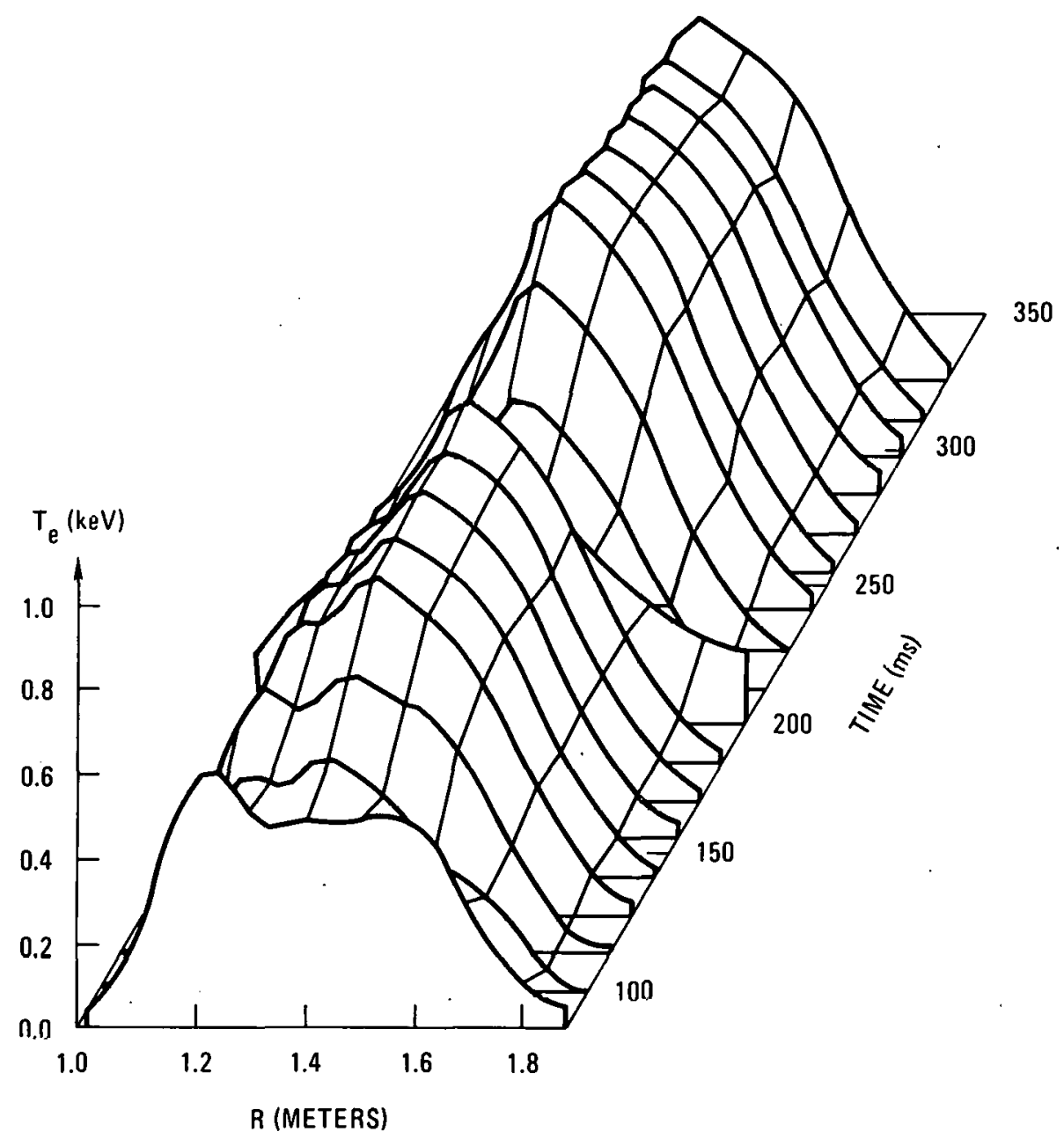

Fig. 7. Electron temperature profile evolution for shot 4890 
In the doublet plasmas the $q$ value on axis is calculated to be 1.0 by a self-consistent determination of the current profile, as will be discussed in Section 6.

Soft $x$-ray measurements also suggest that the energy balance. in the central region of the plasma is not dominated by metallic impurity radiation. A typical x-ray spectrum is shown in Fig. 8. The most prominent lines are molybdenum at $2.7 \mathrm{keV}$ and chromium at $5.5 \mathrm{keV}$. The relative impurity concentration inferred from the soft $x$-ray intensity is about $10^{-4}$. For coronal equilibrium this concentration implies that less than $10 \%$ of the local ohmic input power is radiated by these metals. Hollow temperature profiles characteristic of severe heavy metal radiation were not observed.

The maximum plasma density obtained during the confinement measurements also indicates a relatively high degree of plasma cleanliness. The maximum line-average density obtained at $24 \mathrm{kG}$ is approximately $5 \times 10^{13} \mathrm{~cm}^{-3}$, a value which is comparable (Fig. 9) to the limiting density in other recent circular tokamak experiments, including several operating with titanium gettering [5-10].

Typical discharge parameters are summarized in Table I. The lower range of densities for the droplet and elliptical discharges reflects the limited amount of experimental time devoted to these configurations, rather than any fundamental difference in maximum density relative to doublet. 


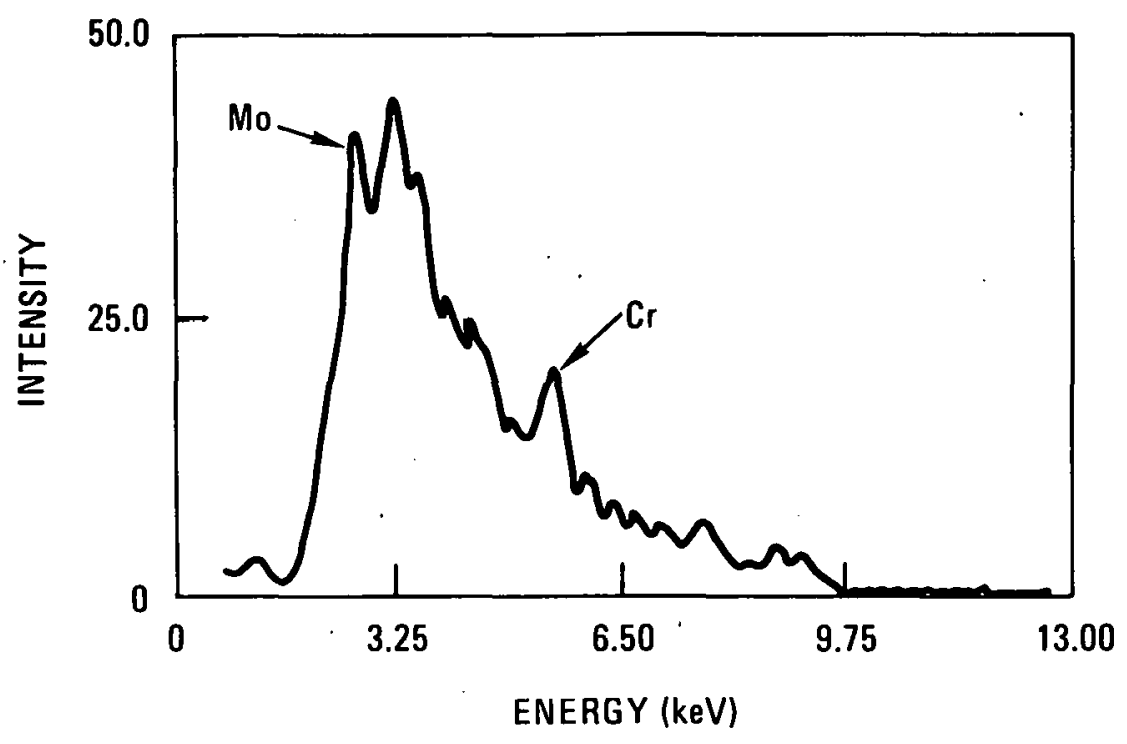

Fig. 8. Typical soft $x$-ray spectrum metallic impurity lines showing

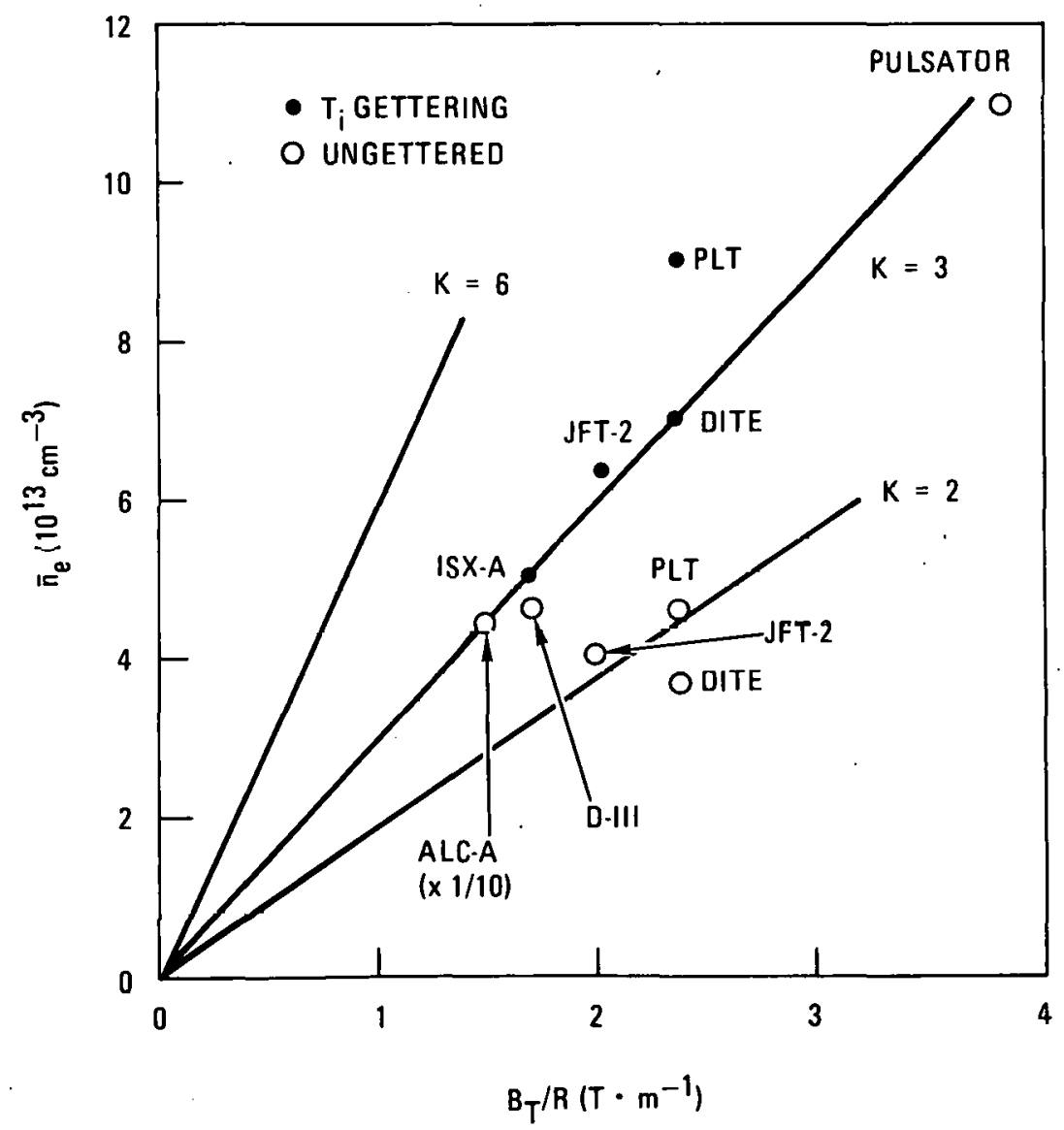

Fig. 9. Comparison of maximum plasma density obtained in Doublet III and other circular tokamaks 
TABLE 1

PLASMA PARAMETERS FOR STEADY-STATE DISCHARGES

\begin{tabular}{|c|c|c|c|}
\hline Parameter & E11ipse & Droplet & Doublet \\
\hline $\mathrm{B}_{\mathrm{T}}(\mathrm{kG})$ & 20 & 20 & 24 \\
\hline Flattop Duration (MS) & 400 & 400 & 400 \\
\hline$I_{p}(k A)$ & 400 & $270(x 2)$ & 1500 \\
\hline Elongation & 1.25 & 1.1 & 3.1 \\
\hline$q(0)$ & $\sim 1$ & 1 & $\sim 1$ \\
\hline Average $\mathrm{q}_{\text {LIMITER }}^{*}$ & 3.9 & 4.3 & 6.9 \\
\hline $\overrightarrow{\mathrm{n}}_{\mathrm{e}}\left(10^{13} \mathrm{~cm}^{-3}\right)$ & $2-3$ & $2.5-3.5$ & $3-5$ \\
\hline $\mathrm{T}_{\mathrm{e}}(0)(\mathrm{keV})$ & $0.7-1.0$ & $0.5-1.0$ & $0.5-1.1$ \\
\hline Minimum Voltage (V) & 1.6 & 1.6 & 1.6 \\
\hline Minimum $z_{\text {eff }}$ & 2 & 1.1 & 1.7 \\
\hline $\mathrm{q}_{\text {LIMITER }}^{*} \equiv$ & & & \\
\hline
\end{tabular}




\section{MAGNETIC ANALYSIS}

The Doublet III magnetic diagnostic system measures the poloidal magnetic flux and field intensity at an array of points surrounding the plasma region. This data can be used to determine the shape and location of the outermost flux surfaces of the plasma, and, in certain cases, can also be used to estimate the current density and safety factor profiles inside the plasma.

Various aspects of the plasma shape and posttion can be computed directly from the magnetic data on a real-time bas1s. The waveforms for the radial position, height and flux fraction inside the doublet separatrix shown in Fig. 6 are obtained in this fashion. The required computations are implemented with analog electronic circuits, the outputs of which are used for feedback control of the plasma position. It has been established empirically that the quantity

$$
P_{s}(m)=2-1.15 \frac{P R 12}{P R 34}
$$

derived from the ratio of the partial Rogowski (PR) magnetic probes on the inside wall approximates the flux fraction inside the separatrix to within $\pm 10 \%$. This diagnostic has proved to be particularily useful in optimizing doublet shaping.

The flux configurations shown in Figs. 4 and 5 are examples of the use of the General Atomic free-boundary MHD equilibrium code GAQ [11] for off-line analysis of the magnetic data. This code solves the GradShafranov equation 


$$
\Delta^{*} \psi=\mu_{0} R j_{\phi}
$$

where $2 \pi \psi$ is the poloidal flux,

$$
\Delta^{*} \equiv R \frac{\partial}{\partial R} \frac{1}{R} \frac{\partial}{\partial R}+\frac{\partial^{2}}{\partial z^{2}},
$$

$R$ and $z$ are the usual toroidal coordinates, and $j_{\phi}$ is the toroidal current density. For purposes of analyzing experimental data, it is convenient (and sufficiently. accurate) to parameterize the toroidal current density as

$$
j_{\phi}=G(\psi)\left[\frac{\beta_{p}}{R_{0}}+\frac{\left(1-\beta_{p}\right) R_{0}}{R}\right] \text {. }
$$

Here $G(\psi)$ is an arbitrary adjustable function (typically a gaussian) that characterizes the flux dependence of the current profile, $R_{0}$ is the major radius, and $\beta_{p}$ is an adjustable constant which characterizes the plasma pressure. The analysis procedure involves solving Eq. (3.1) for a given $G(\psi)$, using the experimentally measured flux values as boundary conditions, and incorporating the constraint that $G(\psi) \equiv 0$ for flux values outside the limiter flux surface. The result of the solution is a plasma configuration which satisfies both MHD equilibrium and the experimentally measured boundary fluxes. Agreement with experiment is tested by comparing the computed and experimentally measured values of the poloidal field at the partial Rogowski locations. The function $G(\psi)$ and the value of $\beta_{p}$ are then adjusted until satisfactory agreement is obtained. A typical result is shown in Fig. 10. For the case of a doublet plasma, a change in the plasma shape corresponding to a $10 \%$ change in the flux fraction inside the separatrix results in an easily detectable change in the poloidal field configuration. 


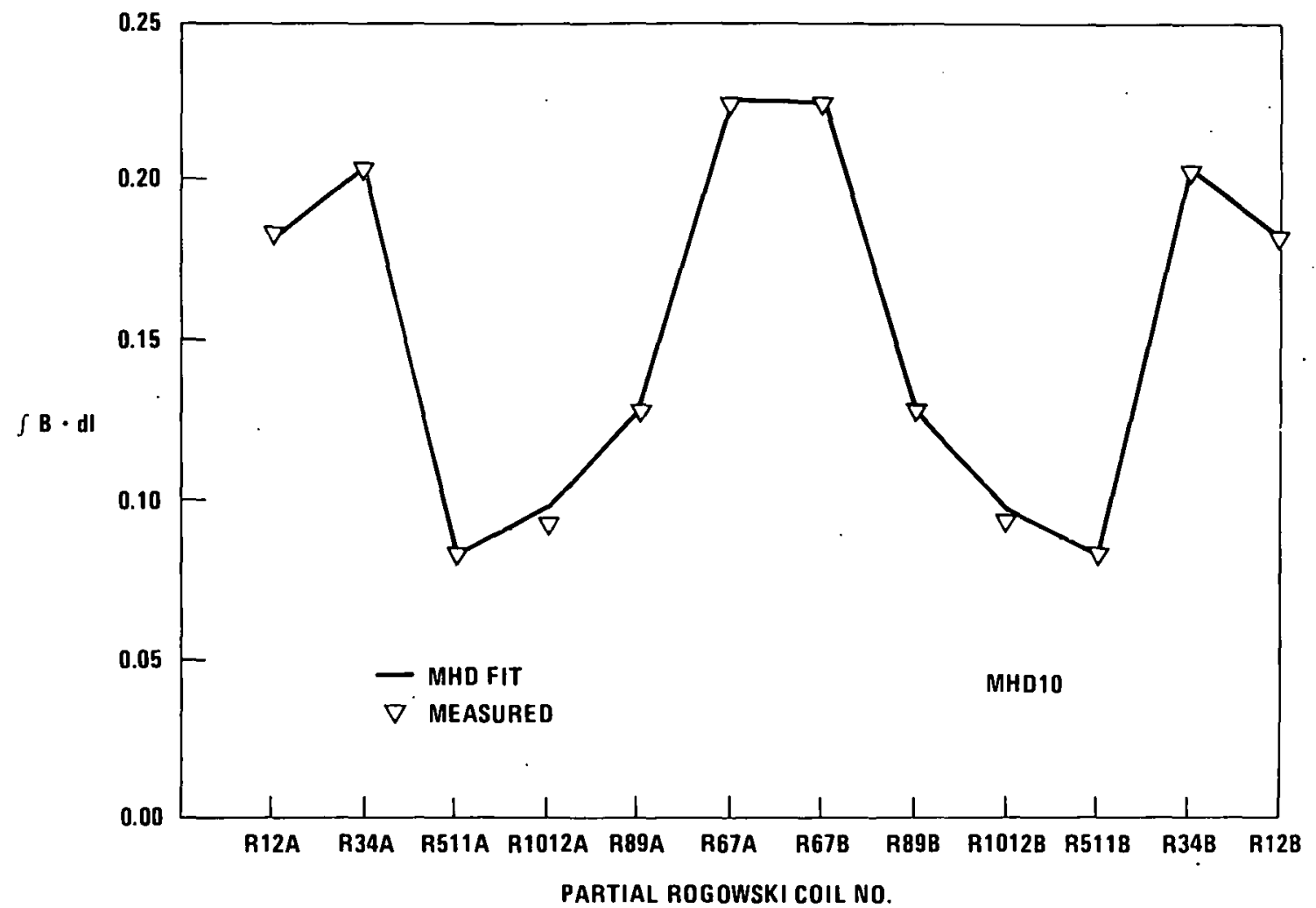

Fig. 10. Comparison of experimentally measured and computed partial Rogowski values 
Strictly speaking, the MHD analysis procedure gives information only about the shape of the outermost flux surfaces of the plasma. However, if $G(\psi)$ is restricted to be a physically reasonable monotonic function of $\psi$ (e.g. gaussian or parabolic to some power), then the internal details of the flux surfaces are relatively insensitive to the exact functional dependence of $G(\psi)$, provided that the degree of peaking of the profile is correct. For plasmas with significant noncircularity, where the shape is sensitive to the current profile, the MHD analysis procedure can provide information about the internal current density and safety factor profiles and also the radial dependence of the flux surface elongation. Figure 11 shows the current profile obtained from the MHD analysis of shot 4890. Elongation data for typical p̈lasmas is shown in Fig. 12. Another example of the use of the MHD analysis procedure to estimate $j(r)$ and $q(r)$ will be given in Section 6 . 


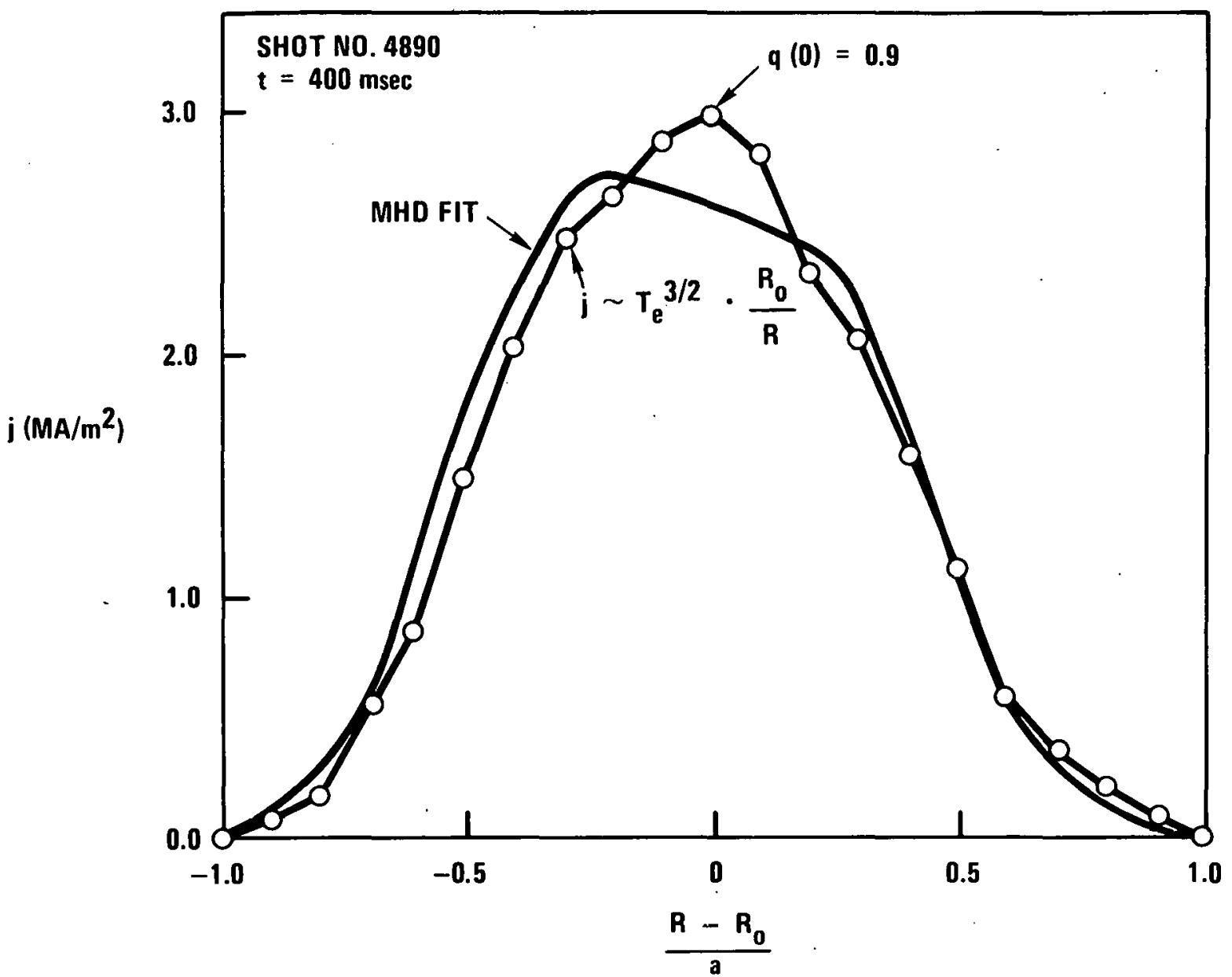

Fig. 11. Current density profile obtained by MHD analysis. The current profile is similar to that expected for Spitzer resistivity and uniform $\mathrm{Z}_{\text {eff }}$. 


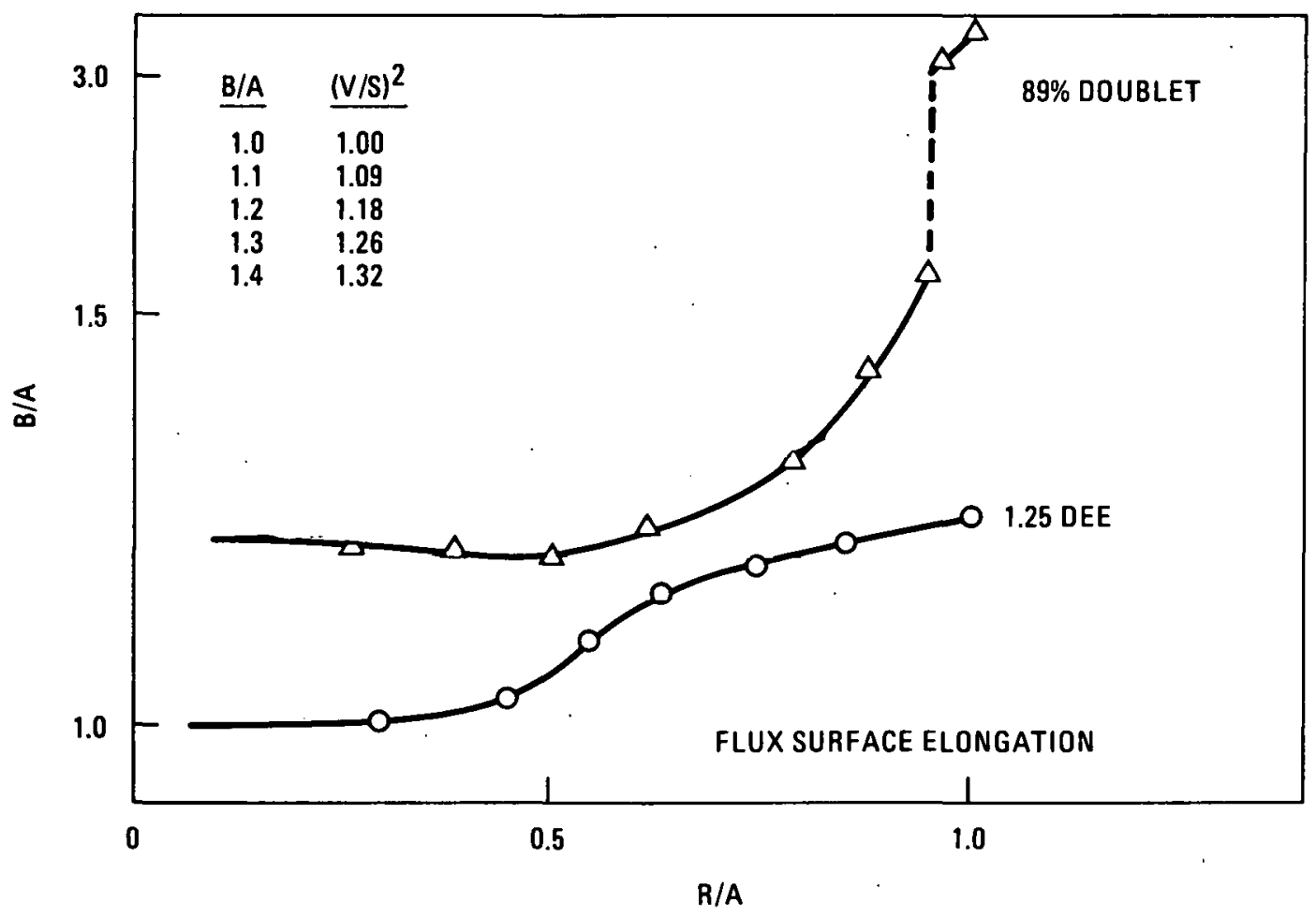

Fig. 12. Flux surface elongation nhtained by MHD analysis 


\section{DENSITY SCALING STUDIES OF ENERGY CONFINEMENT}

The scaling of the central values of electron temperature, electron energy confinement time and $\mathrm{Z}_{\text {eff }}$ has been studied in Doublet III as a function of plasma denșity and configuration. As will be shown in the following section, the central and global values of the electron energy confinement time and $Z_{\text {eff }}$ are nearly equal. (within $\pm 20 \%$ ) for typical plasmas in Doublet III (and in other tokamaks), and hence measurements of the central parameters make it possible to study the scaling properties with a minimum amount of data analysis, and to include data where only central values of plasma parameters were measured.

The central electron energy confinement time is defined as

$$
\tau_{E e^{(0)}} \equiv \frac{\frac{3}{2} n_{e}(0) k T_{e}(0)}{E(0) j(0)}
$$

where $n_{e}(0), T_{e}(0), E(0)$ and $j(0)$ are respectively the on axis values of electron density, electron temperatures, electric field and current density. In steady-state conditions, with the electric field fully penetrated, the electric field on axis is. simply

$$
\mathrm{E}(0)=\mathrm{V} / 2 \pi \mathrm{R}(0),
$$

where $V$ is the loop voltage and $R(0)$ is the major radius. 
In the flattop phase of the discharge where the plasma current, loop voltage, and temperature profile are constant, this approximation is very accurate.

The current density on axis is given by

$$
j(0)=\frac{2 B_{T}}{\mu_{0} R q(0)}\left(\frac{1+\kappa_{0}^{2}}{2 \kappa_{0}}\right),
$$

where $k_{0}$ is the elongation of the central flux surface. Since the central flux surface is nearly circular $(\kappa \leq 1.2)$, the term in parenthesis is essentially unity. Furthermore, if $q(0)=1.0$, then

$$
j(0)=\frac{2 B_{T}}{\mu_{0} R(0)} \text {, }
$$

and calculation of $\tau_{E e}(0)$ is straightforward once the central values of electron density and temperature are known.

The $Z_{\text {eff }}$ value on axis is calculated from the enhancement factor $\alpha$ to Spitzer resistivity in Ohm's law

$$
E(0)=\alpha \eta_{S P}(Z=1) j(0)
$$

where $\alpha$ and $z_{\text {eff }}$ are approximately related by

$$
\alpha=z_{\text {eff }}\left[0.57+\frac{0.90}{z_{\text {eff }}+1.08}\right]
$$

and

$$
\eta_{S P}=\frac{m_{e} e_{i}}{2 n_{e} e^{2}}
$$


The location of the diagnostic measurements is not necessarily coincident with the magnetic center of the plasma, as shown in Fig. 3. Thus to evaluate Eq. (4.1), certain simplifying assumptions are necessary. For the analysis in this section only, we assume that a) the central value of the electron temperature is either the value obtained from the laser scattering or the peak temperature along the $2 \omega_{c e}$ radiometer chord, b) the central value of the density is 1.5 times the line-average value measured by the $2 \mathrm{~mm}$ interferometer, and c) $q(0)=1.0$. The validity of these assumptions is established in Section 5, where it is shown that they typically result in a $25 \%$ underestimate of the true central confinement time $\tau_{E e}(0)$. To avoid confusion in the subsequent discussion we will use the notation * to indicate quantities evaluated using the assumptions given above. Thus $\mathrm{T}_{\mathrm{Ee}}^{*(0)}$ denotes the "central" electron confinement time evaluated using Eq. (4.1) and assumptions (a) $-(c)$.

The axial value of $q$ is known to be unity in droplets from the observation of sawteeth. In doublets, the MHD analysis implies $q(0) \sim 1$, but the absence of sawteeth suggests $q(0)$ may still be slightly above unity. Sawteeth were not observed in the elliptical plasmas, and the MHD analysis does not give a definitive estimate of the axial current density. More recent elliptical data suggests that $q(0)$ may have been in the range 1.0-1.5. If the actual axial $q$ was greater than unity for ellipses or doublets, then using Eq. (4.4) underestimates $\tau_{\mathrm{Ee}}(0)$ and overestimates $Z_{\text {eff }}(0)$ by the same factor.

Other than the above assumptions, the principal uncertainty in the calculations comes from the temperature data. Since the laser measures the temperature at a point about $5 \mathrm{~cm}$ from the magnetic axis of the plasma, the raw laser data consistently underestimates the peak temperature by about $10 \%$. This plasma shift also introduces about $10 \%$ uncertainty in the $2 \omega_{c e}$ radiometer calibration. 'lhe total uncertainty in either temperature measurement is estimated to be about $\pm 20 \%$. The shot-to-shot scatter in the data is approximately $\pm 10 \%$. Overall, the uncertainty in the central 
confinement time is estimated to be about $\pm 30 \%$, and is also subject to the axial $\mathrm{q}$ and density profile assumptions discussed above. Within the data set, the'relative uncertainty is less, but the use of laser data for the droplet and ellipse data and radiometer data for the doublet data could introduce a systematic error of up to $20 \%$. Similarly, uncertainty in $\mathrm{T}_{\mathrm{e}}(0)$ results in a $\pm 30 \%$ uncertainty in $\mathrm{Z}_{\text {eff }}^{*}(0)$.

The range: of electron temperature and $Z_{\text {eff }}$ values obtained in the scaling studies is similar to that observed in PLT for ungettered discharges [5]: The on-axis values of $\mathrm{T}_{e}$ and $\mathrm{Z}_{\text {eff }}$ both decrease as the plasma density is increased with larger gasinjection inputs. Typical values range from $\mathrm{T}_{\mathrm{e}}^{*}(0)=1.1 \mathrm{keV}$ and $\mathrm{Z}_{\mathrm{eff}}^{*}=6$ at $\overline{\mathrm{n}}_{\mathrm{e}}=1.5 \times 10^{13} \mathrm{~cm}^{-3}$ to $\mathrm{T}_{\mathrm{e}}^{*}(0)=0.7 \mathrm{keV}$ and $z_{\text {eff }}^{*}=2$ at $\bar{n}_{e}=4.5 \times 10^{13} \mathrm{~cm}^{-3}$. The value of $z_{\text {eff }}$ decreases approximately as $1 / \bar{n}$. Droplet plasmas with $Z_{\text {eff }}^{*}=1.1$ were obtained, but $Z_{\text {eff }}^{*}=$ 1.5-2 was more typical. Doublet plasmas exhibited a wider range of $\mathrm{Z}$ eff values at a given density, presumably due to a varying degree of plasmawall interaction during the initial phase of the discharge (see Fig. 6). Lower $z_{\text {eff }}$ values were obtained in those discharges which had a lesser degree of configurational variation during the initial phase.

The central electron confinement times for near-circular and doublet discharges are plotted in Fig. 13. The confinement time increases with density, as expected with Alcator scaling. At higher densities, the data fito the ocaling law

$$
\tau_{\mathrm{Ee}}^{*}(0)=\left(3 \times 10^{-19} \mathrm{~cm}-\mathrm{sec}\right) \overline{\mathrm{na}}{ }^{2}
$$

At the higher densities, $\mathrm{n}_{e} \approx \mathrm{n}_{i}$ and $\mathrm{T}_{\mathrm{e}} \approx \mathrm{T}_{i}$ (see Section 7 ), so that the coefficient for the total confinement time would be twice that given above (i.e., $6 \times 10^{-19} \mathrm{~cm}-\mathrm{sec}$ ), which is approximately equal to the value observed in Alcator-A [9].

There is no detectable difference in confinement between the nearcircular and doublet cases. As is discussed in Appendix I, in the low to 


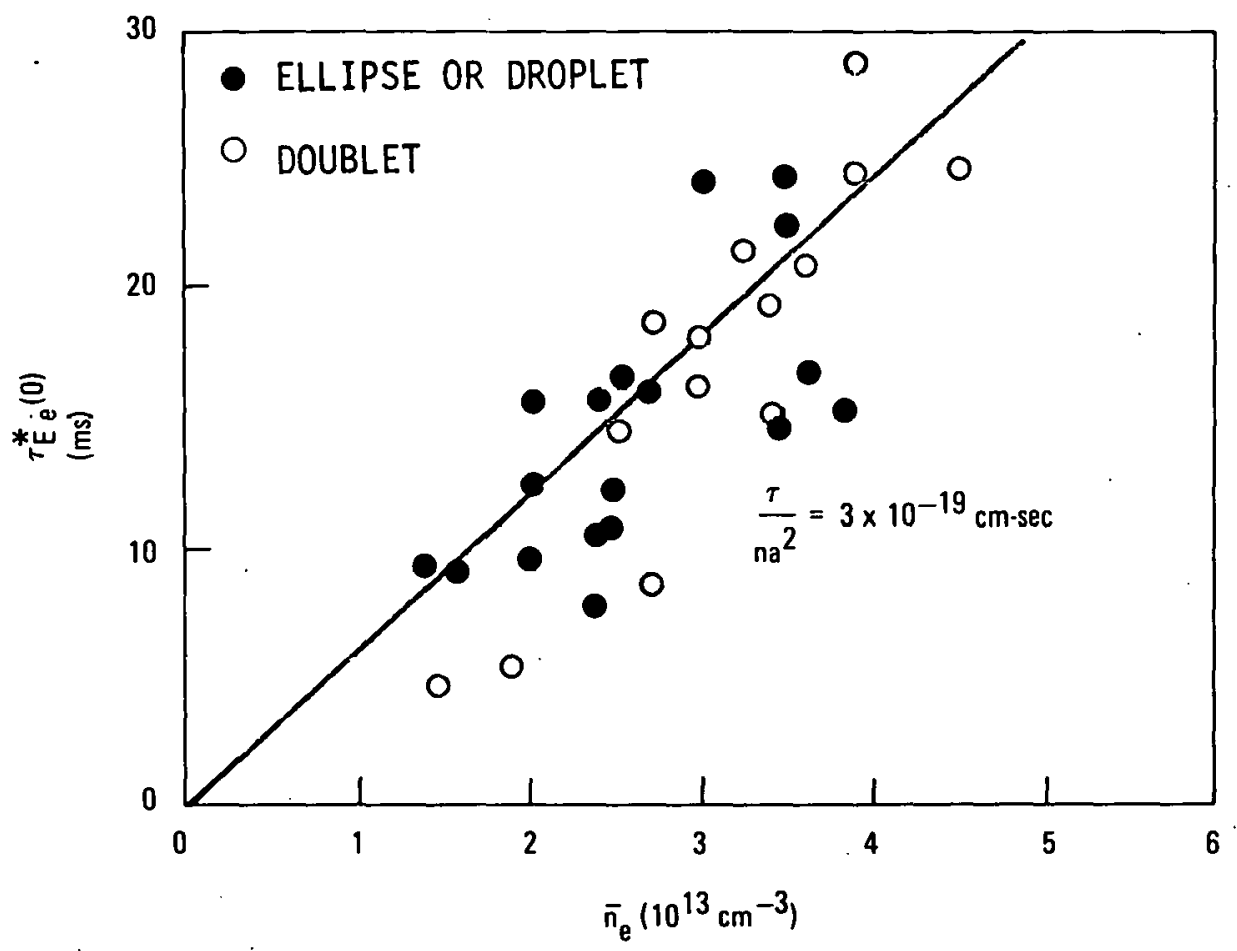

Fig. 13!. Density scaling nf central plectron energy confinement time for near-circular and doublet plasmas 
medium density regime where anomalous electron thermal transport is the dominant energy loss mechanism, the confinement improvement owing to noncircularity is theoretically expected to be

$$
\frac{\tau(k)}{\tau(k=1)}=\frac{2 k^{2}}{1+k^{2}}
$$

where $k$ is the elongation of the flux surfaces. From Fig. 12, the effective elongation of doublets in the central part of the plasma where most of the plasma energy resides is about 1.2, and hence, using Eq. (4.9), the expected confinement improvement for doublet is about 1.18. A difference of this magnitude is not expected to he observable, given the scatter of the data and the other possible systematic errors discussed above. 


\section{GLOBAL ENERGY CONFINEMENT ANALYSIS}

The global energy confinement time for a stationary plasma, defined as

$$
\tau_{E}=\frac{3 / 2 \delta\left(n_{e} k T_{e}+n_{i} k T_{i}\right) d^{3} V}{I V_{L}}
$$

is the most direct measure of the total energy confinement. Here I and $\mathrm{V}_{\mathrm{L}}$ are the plasma current and loop voltage, and the integral is to be evaluated over the plasilu volume, which in principle requires knowledge of the electron and ion temperatures and densities at each point in the plasma. Since actual diagnostic data consists of a limited number of point or lineintegral measurements, simplifying assumptions are required. For circular cross-section plasmas, radial symmetry is generally assumed. For noncircular plasmas, and in particular for doublets, more elaborate methods are necessary.

In Doublet III, the related problems of interpreting diagnostic measurements and determining the p.lasma stored energy have been addressed by develping a gentidil purpose nonc1rcular energy analysis code.

The concept of the energy analysis method is illustrated in Fig. 14. The flux configuration $\psi(R, Z)$ determined by the MHD analysis procedure described in Section 3 serves as the starting point. After $\psi\left(R, Z_{1}\right)$ is determined, numerical, methods are used to transform the experimentally measured data sucli as $\mathrm{l}_{e}(R)$ and $\bar{n}(R)$ into corresponding functions of the flux $\psi$. Once $n(\psi)$ and $T(\psi)$ are known, evaluation of the volume integral of $\mathrm{nT}$ is straightforward. In this.report, only electron lemperature and 


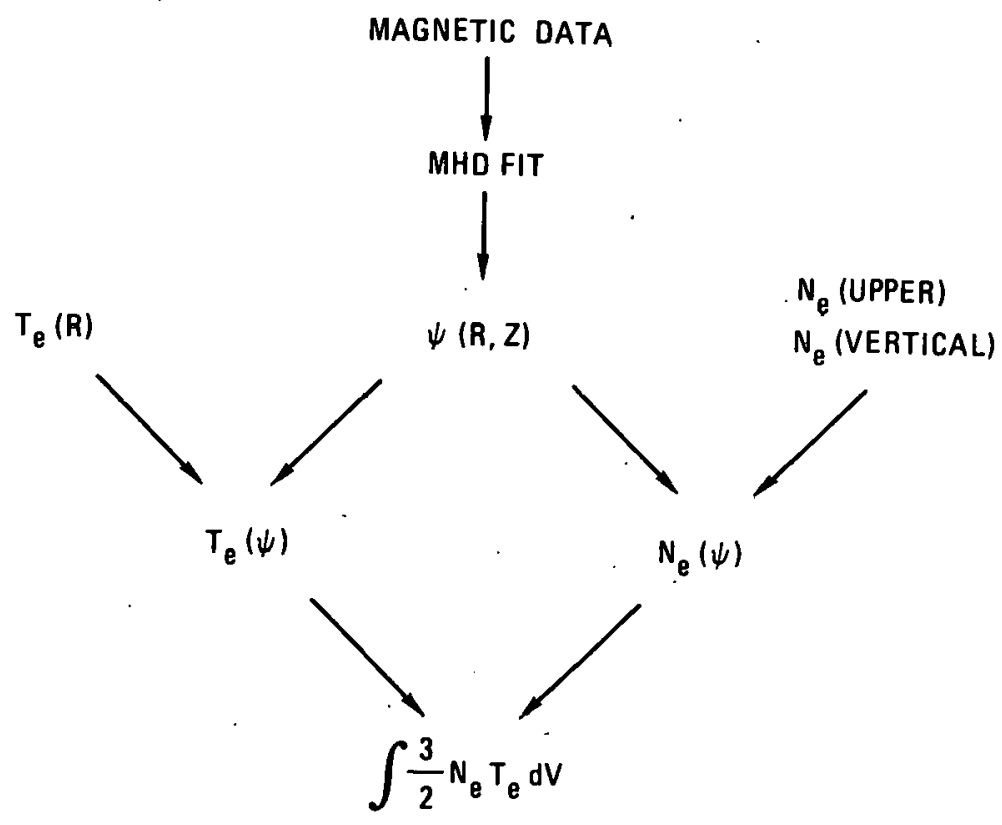

Fig. 14. Electron energy confinement analysis concepl 
density data are analyzed. However, the method can be easily extended to other experimentally measureable quantities.

Further details of the analysis method are described below. First, the magnetic data is analyzed to obtain the plasma flux configuration $\psi(R, Z)$. Subsequent analysis is carried out using a normalized flux coordinate

$$
\tilde{\psi} \equiv \frac{\psi-\psi_{\text {axis }}}{\psi_{\text {limiter }}-\psi_{\text {axis }}}
$$

Typically, the flux is approximately parabolic, so that $\tilde{\psi} \sim r^{2} / a^{2}$. Since the pressure is constant along a flux surface, and hecause of high electron thermal conductivity along a field line, the electron density and temperature are also constant and are functions only of $\tilde{\psi}$. By dirtogy with ctrcular discharges, the density and temperature profiles are assumed to be

$$
\begin{aligned}
\mathrm{n}_{e} & =\left(\mathrm{n}_{e o}-\mathrm{n}_{e, 1 i m}\right)(1-\tilde{\psi})^{\alpha}+\mathrm{n}_{e, 1 \mathrm{im}} \\
\mathrm{T}_{e} & =\left(\mathrm{T}_{e o}-\mathrm{T}_{e, 1 i \mathrm{~m}}\right)(1-\tilde{\psi})^{\alpha}+\mathrm{T}_{e, 1 \mathrm{im}}
\end{aligned}
$$

These functions give an accurate representation of experimental data. If hollow profi.les had been observed, a different form would have heen necessary. Each function has three adjustable coefficients, the peak value, the limiter value, and $\alpha$, the exponent of $(1-\tilde{\psi})$. The temperature coefficients are found by a least squares fit to point measurements of temperature along the chord viewed by the radiometer. Each spatial point in the radiometer profile has a corresponding fiux value used when comparing the temperature function to the data. The density coefficients are determined by a similar procedure, except that line integrals as well as point measurements are involved. A more complicated numerical algorithm compares the measured 
values with integrals computed using the density function. In this approach, the true path length through the plasma is evaluated. Finally, the functions determine the values of electron density and temperature at every point in the plasma, and the stored energy integral is evaluated.

The energy analysis method has been applied to several doublet plasmas. Representative results for a 90\% doublet (\$4890) are shown in Figs. 15 and 16. Figure 15 shows the radiometer data and the corresponding fitted function

$$
\mathrm{T}_{\mathrm{e}}=940 \mathrm{eV}[1-\tilde{\psi}]^{1.50}
$$

The density profile is

$$
\mathrm{n}_{\mathrm{e}}=6.1 \times 10^{13} \mathrm{~cm}^{-3}[1-\tilde{\psi}]^{0.82} .
$$

The density profile along the upper $2-\mathrm{mm}$ microwave chord at $Z=90 \mathrm{~cm}$ is shown in Fig. 16. For comparison, the measured line-average density is $3.8 \times 10^{13} \mathrm{~cm}^{-3}$. The total electron stored energy is $46 \mathrm{~kJ}$. The ohmic power is $2.5 \mathrm{MW}$, resulting in a global electron energy confinement time of $19 \mathrm{msec}$.

For comparison, the true central confinement time obtained using Eqs. 5.5 and 5.6 and $q(0)=1.1$ is $32 \mathrm{msec}$. The central parameter analysis technique used in Section 4 gives $\tau_{\mathrm{Ee}}^{*}(0)=24 \mathrm{msec}$. The global time is found to be $79 \%$ of $\tau_{\mathrm{Ee}}^{*}(0)$ estimated without knowledge of the profiles.

Other than the fundamental uncertainti,es in the diagnostic measurcments themselves, the primary uncertainty in the confinement analysis comes from the procedure used to obtain the density profile. In essence, the density profile form factor $\alpha_{n}$ is determined by the ratio of the horizontal path and vertical pather interferometers. The value of $\alpha_{n}$ is quite sensitive to this interferometer ratio, which was $0.8 \pm 0.1$ for typical $90 \%$ 


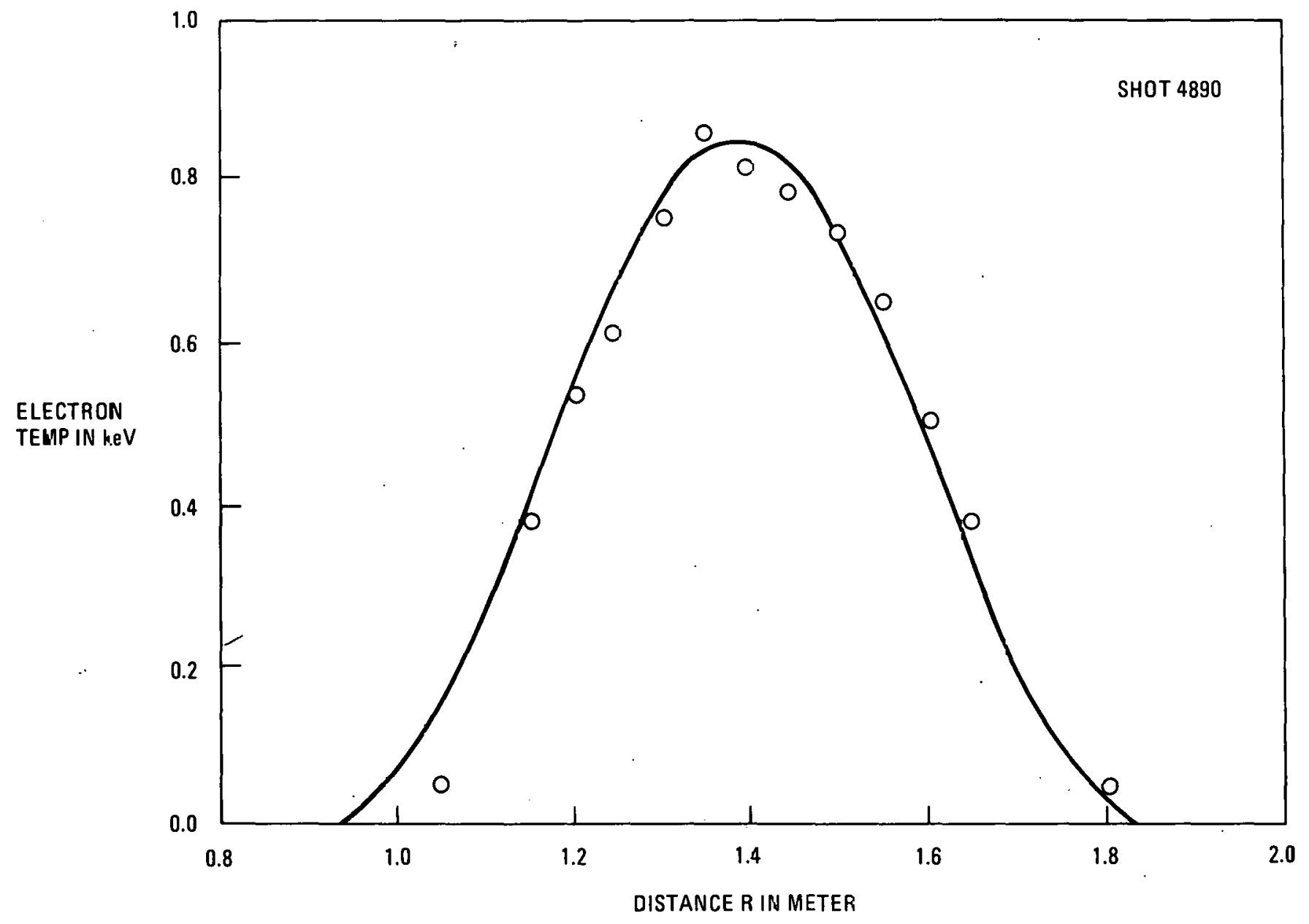

Fig. 15. Comparison of flux function temperature profile and radiometer data 


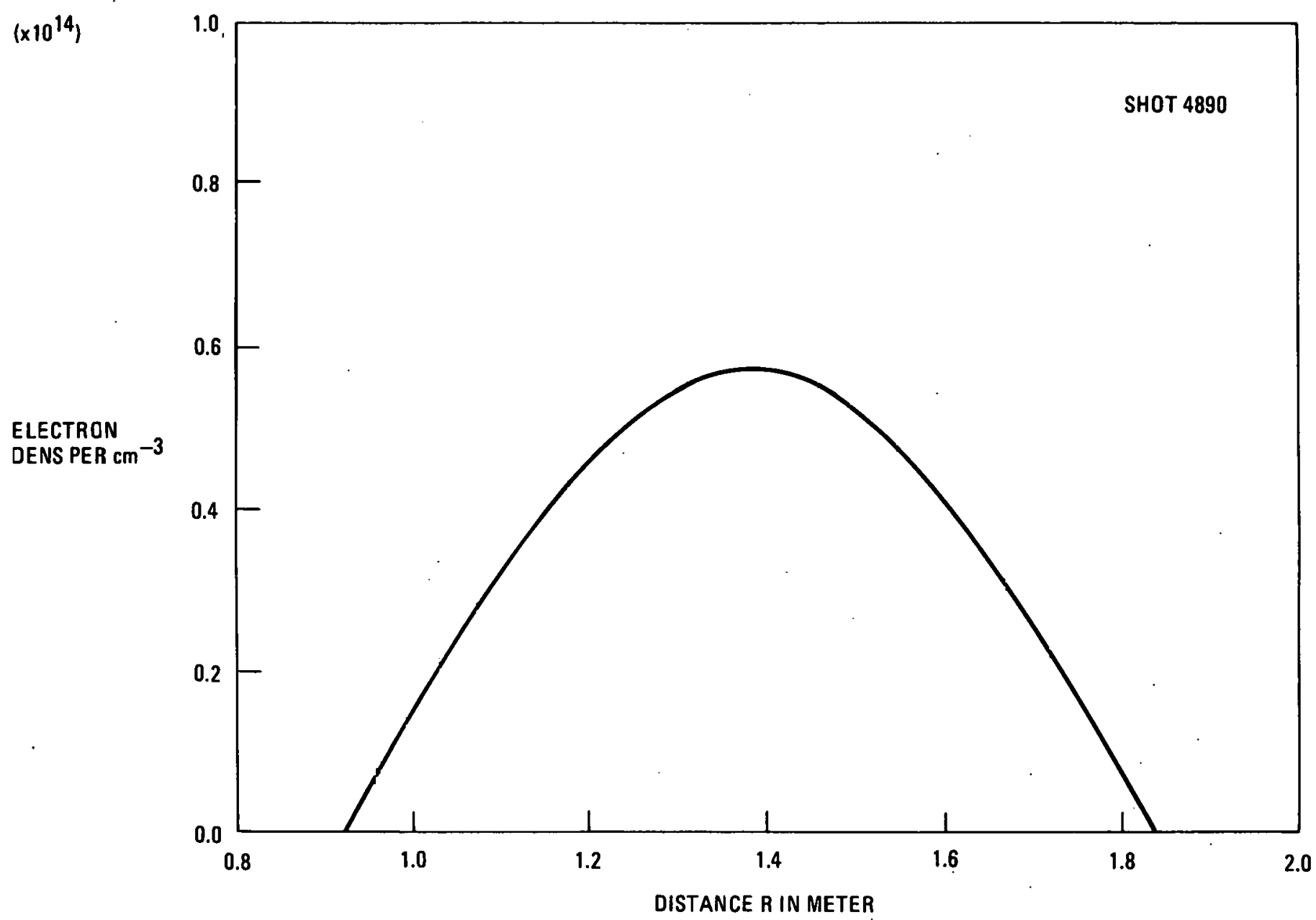

Fig. 16. Calculated density profile 
doublets (Fig. 17). However, there is some scatter in the data, and it is not possible to completely rule out profiles with $0 \leq \alpha_{n} \leq 2$. Future measurement of doublets in which reliable midplane interferometer data is available will narrow the range of possible $\alpha_{n}$ values.

In spite of the lack of complete knowledge of the density profile, the resultant uncertainty in the global confinement time is small. If the same line average density is maintained, the total plasma energy is quite insensitive to the density profile, and the change in $\tau_{E e}$ due to the possible variation in $\alpha_{n}$ is less than $15 \%$.

Another possible source of error comes from the use of an ad hoc model for the plasma current and pressure profiles (Eq. 3.2) in calculating the original MHD equilibrium. Extensive studies have shown, however, that the ad hoc model provides an accurate fit to experimental data, and that minor changes in the flux configuration have a negligible effect $(<10 \%)$ on the stored energy calculation. Overall, the total uncertainty in $\tau_{E e}$ due to the energy analysis procedure is estimated to be less than $20 \%$. This uncertainty is independent of the fundamental uncertainties inherent in the calibrations of the individual diagnostics, as discussed in section 4. Note, however, that no assumption about $q(0)$ is required. 


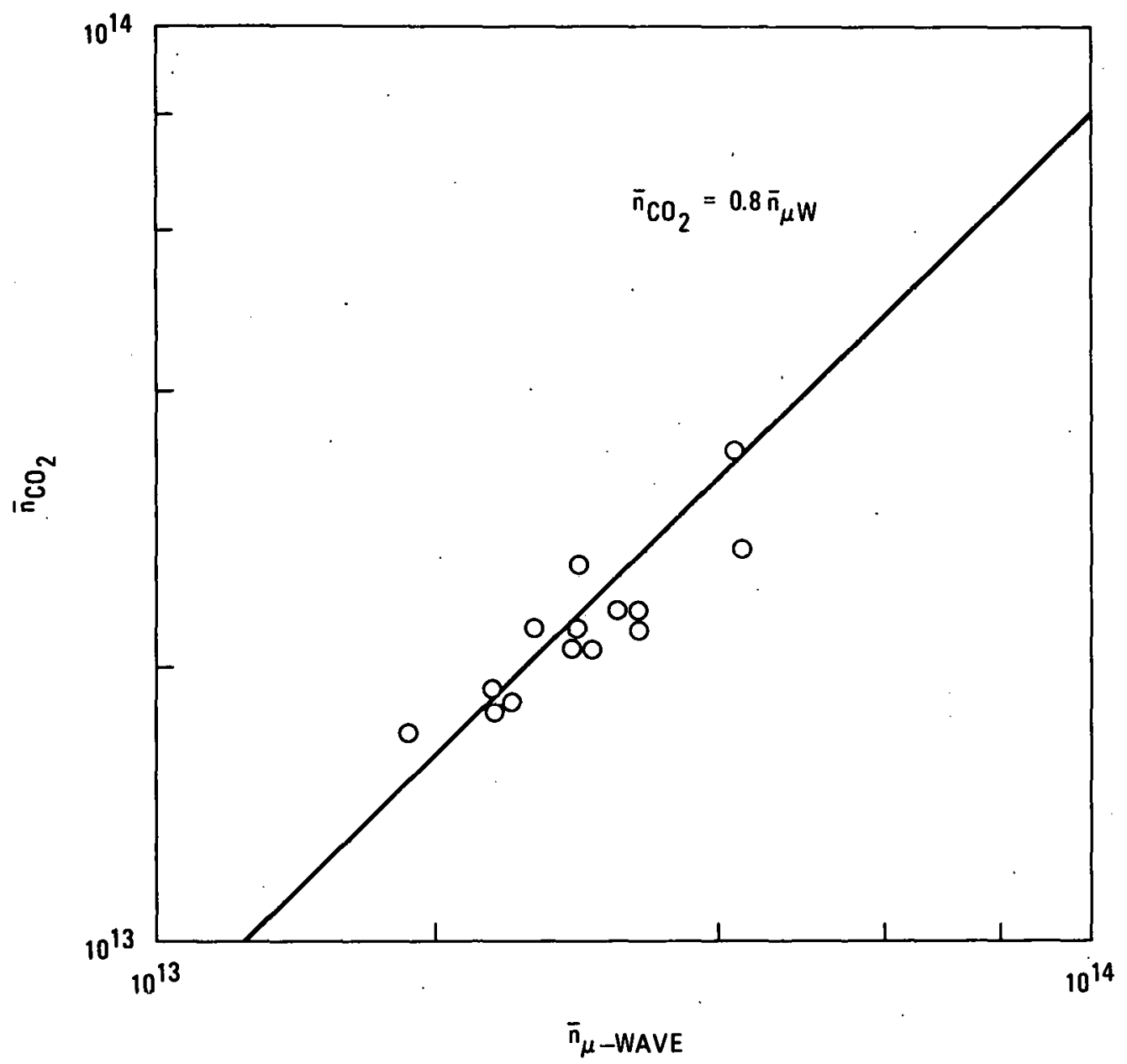

Fig. 17. Interferometer data for $90 \%$ doublets 


\section{SELF-CONSISTENT MHD AND ENERGY ANALYSIS}

The energy analysis algorithm described in the previous section provides the means to carry out a completely self-consistent calculation of the plasma configuration and profiles. The self-consistent procedure replaces the ad hoc assumptions introduced in the MHD analysis with more fundamental physics, and thus eliminates a potential source of uncertainty. In addition to confirming the energy analysis obtained with the ad hoc model, the self-consistent procedure also makes it possible to determine the 7 eff profile in noncircular plasmas.

The basic idea in the self-consistent procedure is to use the plasma temperature and density profiles determined in the energy analysis to explicitly calculate the source term for the Grad-Shafranov equilibrium equation (Eq. 3.1). Instead of using Eq. (3.2), the toroidal current density $j_{\phi}$ is calculated from

$$
j_{\phi}=-R p^{\prime}(\psi)-\frac{f f^{\prime}(\psi)}{\mu_{0} R}
$$

where $f \equiv \mathrm{RB}_{\mathrm{T}}$ and ' denotes the derivative with respect to $\psi$. The value of $\mathrm{p}^{\prime}$ is obtained using

$$
p^{\prime}(\psi)=\frac{d}{d \psi}\left(n_{\underline{i}} k T_{i}+n_{e} k T\right)
$$

where the ion temperature and density are assumed to be

$$
\mathrm{n}_{i}=\mathrm{C}_{\mathrm{i}} \mathrm{n}_{\mathrm{e}}
$$

and

$$
\mathrm{T}_{\mathbf{i}}=\mathrm{C}_{\mathrm{T}} \mathrm{T}_{\mathrm{e}}
$$


In the present analysis $C_{i}$ and $C_{T}$ are taken to be unity. In future analysis, the ion temperature and density profiles can be explicitly included once the appropriate data is available.

The function $\mathrm{ff}^{\prime}$ is calculated using

$$
f^{\prime}(\psi)=\frac{-\mu_{0}}{\left\langle_{R^{-2}}\right\rangle}\left[\langle j / R\rangle+p^{-}(\psi)\right],
$$

where < > denotes the flux surface average, and

$$
\langle j / R\rangle=\frac{1}{2 \pi \eta}\left\langle R^{-2}\right\rangle v
$$

The plasma resitivity $n$ is taken to be

$$
\eta=\eta_{S P} Z_{\text {eff }}(\psi)
$$

where $Z_{\text {eff }}(\psi)$ is given by

$$
z_{\text {eff }}=z_{0}\left[\left(1-c_{z}\right)(1-\tilde{\psi})^{\alpha} z+c_{z}\right]
$$

Here, $Z_{0}, C_{Z}$ and $\alpha_{Z}$ are adjustable constants that specify the $Z_{\text {eff }}$ profile. For given values of $C_{Z}$ and $\alpha_{Z}$, the value of $Z_{0}$ " 1 s chosen such that the total current calculated by integrating $\langle j / R\rangle$ over the plasma area gives the experimentally measured plasma current.

The self-consistent computational procedure begins by using the method of Section 5 to determine $n(\psi)$ and $T(\psi)$. Initial estimates for the coefficients $C_{Z}$ and $\alpha_{Z}$ are introduced, and $\mathrm{P}^{\prime}$ and $\mathrm{ff}^{\prime}$ are calculated. Using Eqs. (6.2-6.6) the resultant Grad-Shafranov equilibrium equation is then 
solved to obtain a new $\psi$ function. The procedure is repealed until a converged solution is obtained. The accuracy of the solution is tested by comparing the computed and partial Rogowski values. The cuefficients $\mathrm{C}_{Z}$ and $\alpha_{Z}$ are adjusted until satisfactory agreement is obtained. A complete solution requires about 2-3 minutes of time on the Magnetic Fusion Energy Cunputer lenter Cray computer, and typically can be completed in about? hours of real time. Typical results of the self-consistent analysis are illustrated in Figs. 18,:19 and 20. The final equilibrium is essentially. identical to Fig. 5. The comparison of experimental to calculated values of partial Rogowski probes is shown. in Fig. 18. The current density is plottcd in Fig. 19 as a function of major radius along the chord measured by the radiometer (any path or point could have been chosen). In Fig. 20, the safety factor $q$ and $Z_{\text {eff }}$ are plotted along the same path. At the. center, the values are $\mathrm{Z}_{\text {eff }}=2.8$ and $\mathrm{q}=1.1$. The $\mathrm{q}$ profile artially goes to infinity at the separatrix, which is near the plasma edge, then decreases * to 8.6 at the plasma edge. The edge value is strongly dependent on the precise location of the separatrix. The central electron energy confinement time is 32 milliseconds, which is $68 \%$ greater than the global electron energy confinement time of 19 milliseconds.

The $Z_{\text {eff }}$ profile is physically reasonable. Forthcoming soft $x$-ray and vacuum ultraviolet spectroscopy measurements will provide alternate means of measuring this important plasma profile. 


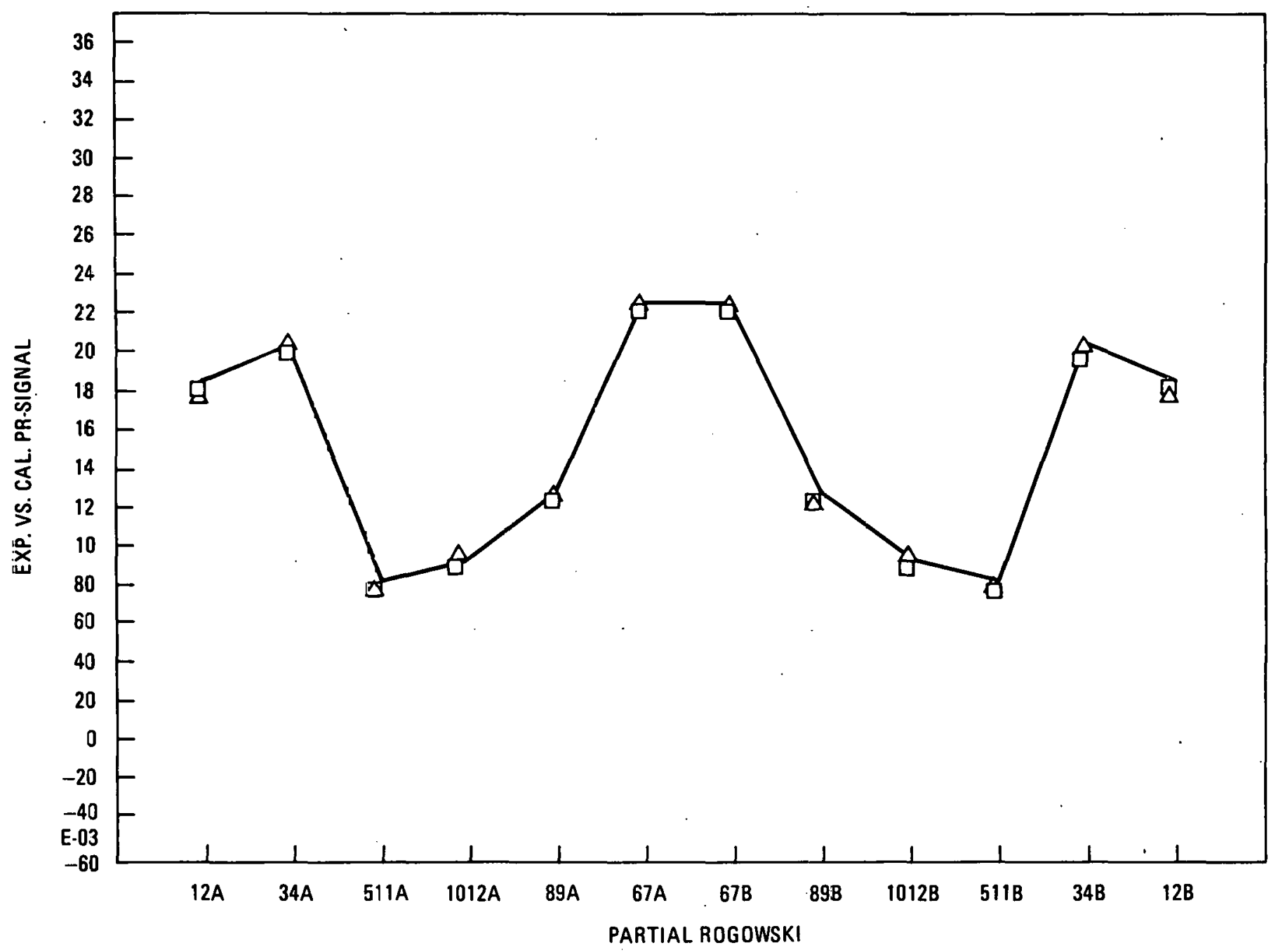

Fig. 18. Somparison of measured and calculated partial Rogowski signals for self-consistent MHD equilibrium 


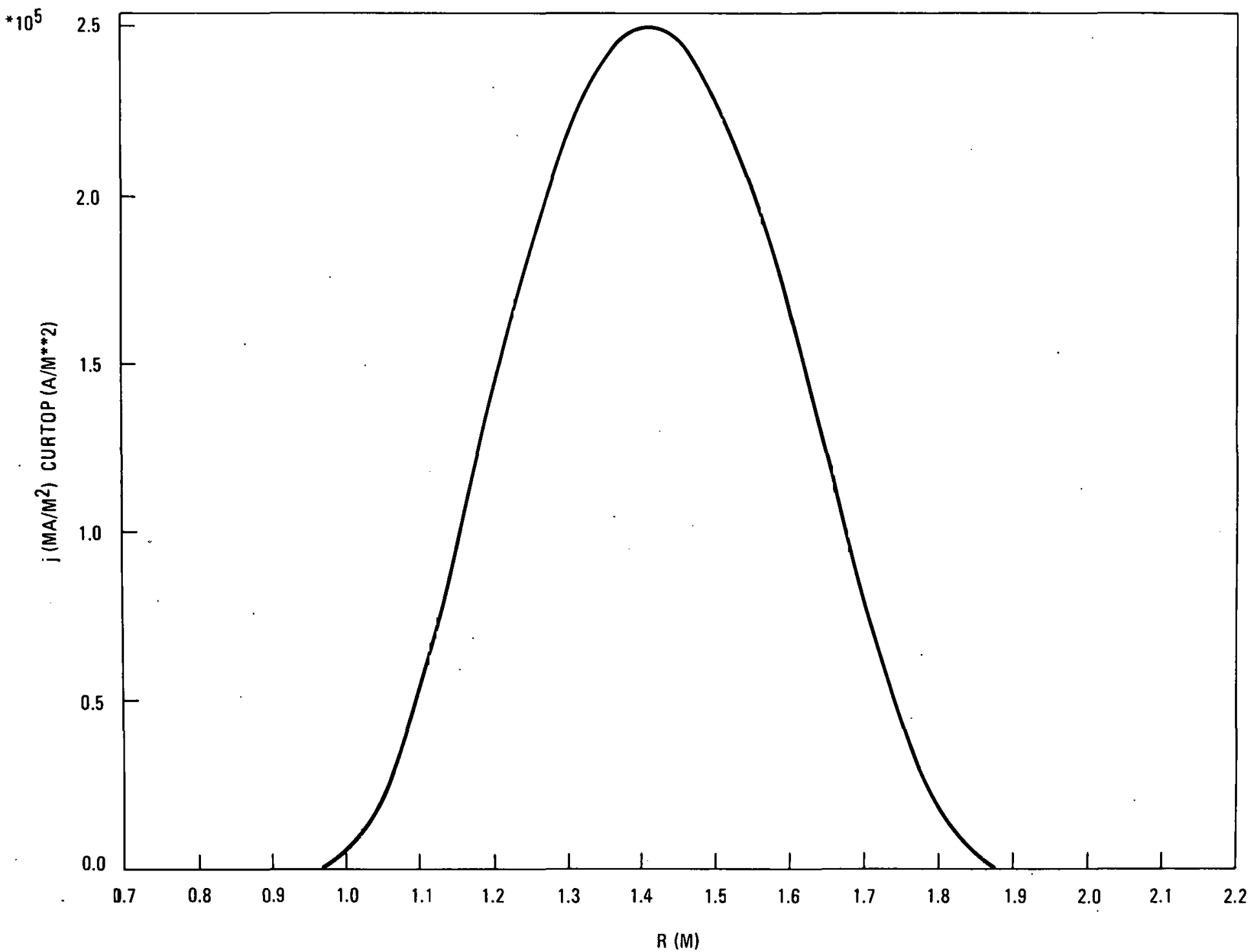

Fig. 19. Current density profile along upper horizontal chord from self-consistent anälysis 


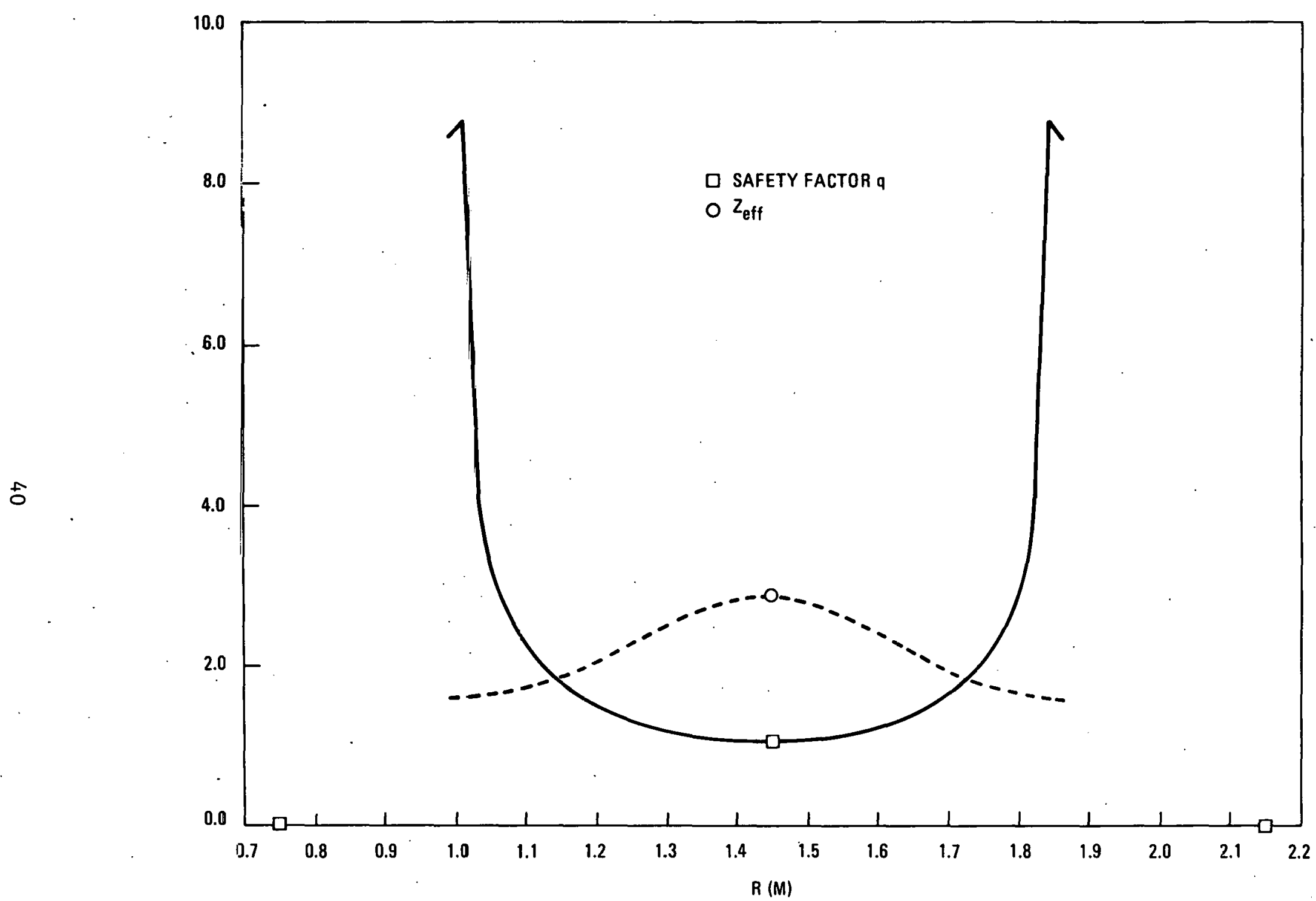

Fig. 20. Safety factor and $Z_{\text {eff }}$ profiles along the upper horizontal chord 


\section{TRANSPORT CODE SIMULATIONS}

The General Atomic 1-1/2-D transport code [12] is used to provide additional information about experimental discharges. Specifically, since ion temperatures are not yet directly measured, they may be inferred from the code. Also, empirical scaling for electrons, $x_{e} \sim 1 / n$, may be verified locally on each flux surface. The code uses a geometrical coordinate $\rho$, proposed by Hinton and Hazeltine [13], analogous to the radial coordinate in the circular case, which is defined at a given point to be proportional to the square root of the toroidal flux $\Phi$ enclosed by the flux surface passing through the point.

$$
\rho \equiv \sqrt{\frac{\Phi}{\pi B_{T}}(0)}
$$

The flux surfaces are determined from a calculated 2-D MHD equilibrium, such as the one shown in Fig. 5. Formulating the transport equations in terms of $\rho$ reduces the problem to a one-dimensiona1. calculation.

The scaling studies described in Section 4 suggest that anomalous electron transport accounts for most of the energy loss in high-density Doublet III plasmas. In the absence of particle transport or charge exchange loss data, the transport is modeled by assuming an anomalous local electron thermal conductivity $x_{e}=\mathrm{K} / \mathrm{n}_{e}$ and standard neoclassical ion thermal conductivity. Particle transport and charge exchange terms are set to zero. Separate calrulations have confirmed that the results are not sensitive to. the neglect of convection. The principal impurity is assumed to be oxygen. The best $\mathrm{fit}$ to the experimental data is obtained for $\mathrm{n}_{\mathrm{e}} \mathrm{x}_{\mathrm{e}}=1.15 \times 10^{18} / \mathrm{cm}-$ sec and $z_{\text {eff }}=2.6$ ( $~ 5 \%$ oxygen). The calculated and experimentally measured values of the loop voltage, on-axis electron temperature and safety factor are in excellent agreement. 
Figure 21 compares the electron temperature profile obtained using the energy analysis method of Section 5 with the profile calculated with the $1-1 / 2-D$ code. The profile is plotted as a function of $(\tilde{\psi})^{1 / 2}$, rough1y proportional to $\mathrm{r} / \mathrm{a}$. The close agreement of the profiles confirms the validity of the assumption of $x \sim 1 / n_{e}$, and also verfies the confinement time calculated in the energy analysis procedure.

The code calculation also predicts that the peak ion temperature is $920 \mathrm{eV}$, nearly equal to the peak electron temperature of $940 \mathrm{eV}$. While the actual value of the ion temperature will be somewhat lower due to charge exchange 1 nsses, the code calculation. suggests that the assumption of equal electron and ion temperatures used in the self-consistent profile analyses is reasonably, valid. Also, as has been mentioned before, the total energy confinement times are expected to be approximately twice the values measured for the electrons alone. 


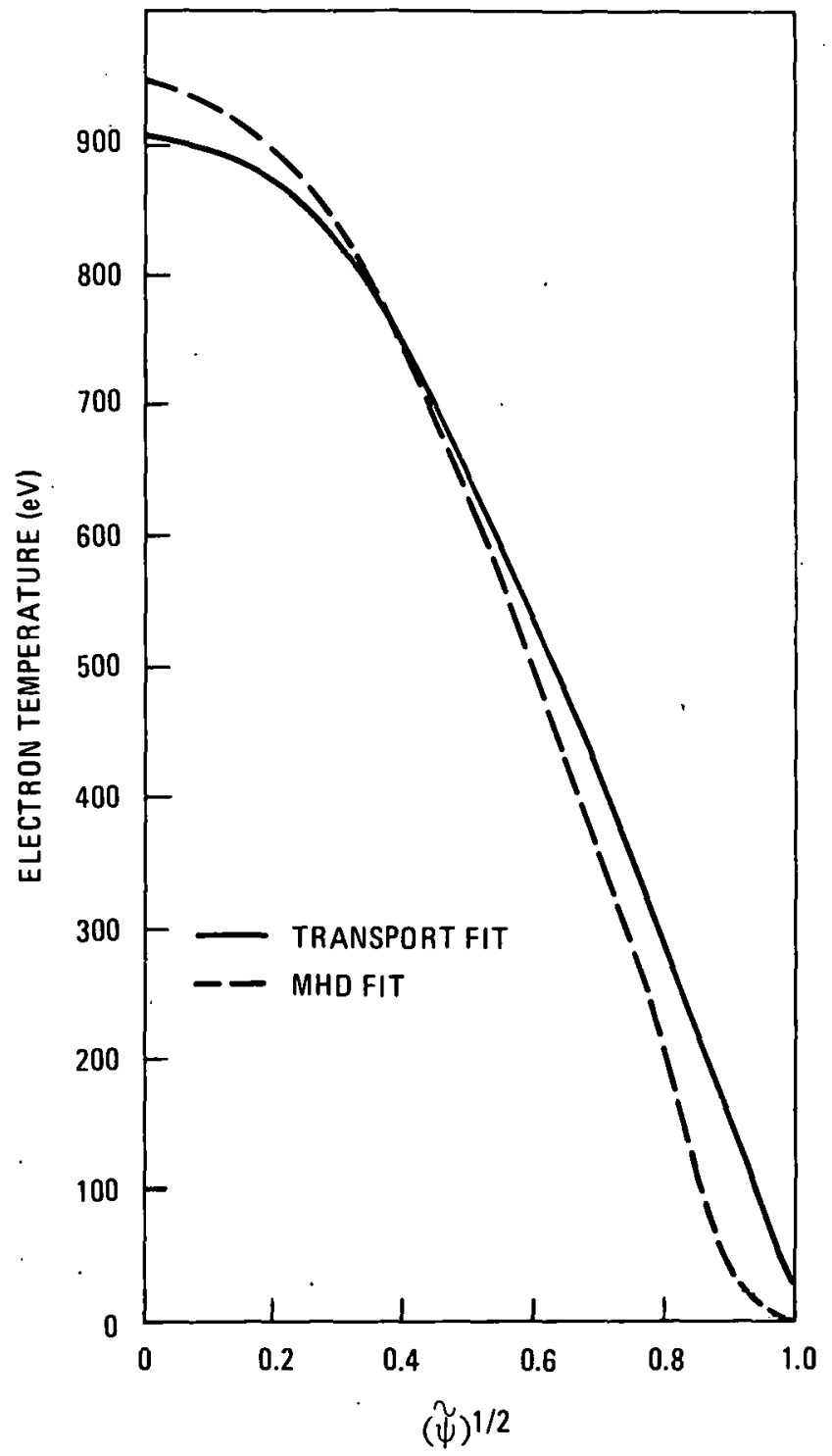

Fig. 21. Comparison of temperature profiles calculated by the MHD-energy analysis and 1-1/2-D transport codes 


\section{SUMMARY AND DISCUSSION}

The electron energy confinement in Doublet III plasmas is found to be equal at a given density for both near-circular and doublet plasma crosssections, and to increase in proportion to the plasma density with an Alcator scaling coefficient similar to that obtained in other circular tokamaks. No deterioration due to the effects of plasma shaping is observed. The lack of any detectable improvement in the confinement of doublets relative to circular plasmas can be understood from magnetic data that shows that the internal flux surfaces of the doublets are only slightly noncircular, and hence that the theoretically expected confinement improvement is less than the $30 \%$ accuracy of the measurements.

Powerful numerical methods for interpreting noncircular diagnostics data and calculating the confinement properties of noncircular plasmas have been developed and exercised to analyze the initial Doublet III data. Consistent results are obtained using a variety of analysis methods, verifying the accuracy of both the experimental data and the analysis procedures. Coupled MHD equilibrium and plasma analysis algorithms make it possible to carry out completely self-consistent calculations of the plasma shape and profiles. The methods developed for confinement analysis can be readily extended to interpret forthcoming ion temperature and radiated power measurements for noncircular plasmas.

At the present time, the highly peaked current profiles inherent with ohmic heating make it Impossible to obtain plasmas with signif1cant noncircularity, either in Doublet III or any other tokamak. Achievement of increases noncircularity will require broadening of the current profile. If such profile control is possible, then the confinement improvement theoretically predicted for noncircular plasmas will be much more readily 
detectable. The analysis techniques described in this report should make confirmation of the improvement possible once the required plasmas are obtained. 


\section{9: : REFERENCES}

[1] T. Ohkawa, "Shaping and Characteristics of Doublet Plasmas in Doublet III," (General Atomic Company Report GA-A15671), 9th European Conference on Controlled Fusion and Plasma Physics, Oxford, 1979.

[2] M. M. Sabado, F. B. Marcus, P. W. Trester and J. C. Wesley, "Doublet III Limiter Performance and Implications for Mechanical Design and Material Selection for Future Limiters," General Atomic Company Report GA-A15633, Oct. 1979. (Presented at the 8th Symposium on Engineering Problems of Fusion Research, San Francisco, 1979).

[3] L. Oren and R. J. Taylor, Nuclear Fusion 17, 1143 (1977).

[4] F. B. Marcus, "Impurity Studies and Discharge Cleaning in Doublet III," General Atomic Company Report GA-A15613, Oct. 1979. (Presented at the IAEA Technical Committee Meeting on Impurities in Tokamaks: Source, Effect, and Control, Alushta, U.S.S.R., October, 1979).

[5] K. Bol, et al., in Plasma Physics and Controlled Nuclear Fusion Research (Proc. 7th Int1. Conf., Innsbruck, 1978), IAEA-CN-37-A1, Vienna: (1979).

[6] M. Murakami, et al., Phys. Rev. Lett. $\underline{42}, 655$ (1979).

[7] S. Konoshima, et al., Journal Nuclear Materials 76-77, 581 (1978).

[8] S. J. Fielding, et al.. Nuctear fusion 17, 1382 (1977).

[9] E. Apgar, et al., in Plasma Physics and Controlled Nuclear Fusion Research (Proc. 6th Int1. Conf., Berchtesgaden, 1976), IAEA-CN-35-A5, Vienna (1977).

[10] W. Engelhardt, et al., in Plasma Physics and Controlled Nuclear Fusion Research (Proc. 7th Int1. Conf., Innsbruck, 1978), IAEA-CN-37-A5, Vienna (1979).

[11] F. W. McClain and B. B. Brown, "GAQ, A Computer Program to Find and Analyze Axisymmetric MHD Plasma Equilibria," General Atomic Report GA-A14490, August, 1977. 
[12] R. L. Miller, "Shape Control of Doublets," General Atomic Company Report GA-A15186, November 1978, Nuclear Fusion 20, 133 (1980).

[13] F. L. Hinton, R. D. Hazeltine, Rev. Mod. Physics 48, 239 (1976).

[14] E. K. Mashke, Plasma Physics, 14, 141 (1972).

[15] K. T. Tsang, Physics of Fluids 20, 1680 (1977). 


\section{Appendix I \\ EFFECTS OF NON-CIRCULARITY ON ENERGY CONFINEMENT}

Noncircular plasmas are theoretically predicted to offer improved confinement. One method of comparing noncircular to circular plasmas is to maintain the same major radius, minor horizontal semi-axis, and central toroidal field, while varying the height to width ratio $k$. As $\kappa$ increases, the geometry of the nested flux surfaces becomes increasingly noncircular. Simultaneousiy, if the limiter value of the safety factor q (inverse rotational transform) is held fixed, the plasma current and poloidal field strength also increase with increasing $k$. Each of these effects results in improved confinement.

First, elongation of the flux surfaces has the immediate geometrical effect of increasing the effective diffusion length for energy transport. Thus, for a given value of the diffusion coefficient $x$, the confinement time is increased. The improvement factor scales as the square of the volume-to-surface ratio. For an ellipse with elongation $\kappa$, the improvement relative to a circle is

$$
\frac{\tau(k)}{\tau(k=1)}=\frac{(V / s)^{2}(k)}{(V / s)^{2}(k=1)}=\frac{2 \kappa^{2}}{1+k^{2}}
$$

This formula is directly applicable if the flux surfaces are uniformly nested ell1pses with constant $k$. For actual plasmas where the flux surface geometry is less ideal, numerical methods for calculating the local gradient length are available. Such methods form the basis of the noncircular transport code described in Section 7, and can calculate the energy 
transport for arbitrary flux geometries. However, in many cases, Eq. (A1) can be used to estimate the degree of confinement improvement by using a value of $k$ characteristic of the average ellipticity of the flux surfaces.

Second, the increase in the poloidal field with increasing $k$ results in reduced ion neoclassical transport. For a simple ellipse the effect is calculated $[14,15]$ to be

$$
x^{\mathrm{NEO}}(\kappa)=\left(\frac{2}{1+\kappa^{2}}\right) x^{\mathrm{NEO}}(\kappa=1)
$$

Again, for less ideal geometrles, numerical methods are available.

The net effect of non-circularity on energy confinement depends on whether electron or ion conduction dominates the energy balance. At low to moderate plasma densities, the energy loss is dominated by the electron transport, which has empirically been found to depend only on the plasma density, with a diffusion coefficient $x_{e}$ given by

$$
x_{e} \sim \frac{5 \times 10^{17} \mathrm{~cm}-\mathrm{sec}}{n_{e}}
$$

In this anomalous electron dominated regime, the improvement due to noncircularity is described by Eq. (A.1).

Because of the $1 / \mathfrak{n}$ dependence of the anomalous electron transport, at a high enough plasma density, ion neoclassical transport becomes the dominant energy loss mechanism. In this regime, both Eqs. (A.1) and (A.2) apply, and the overall improvement with elongation becomes

$$
\frac{\tau(\kappa)}{\tau(\kappa=1)}=k^{2}
$$


Since the electron loss scales as $1 / n$ and the ion loss scales as $n$, the total energy confinement time will reach a maximum at some intermediate density. The density at which the maximum occurs and the relative value of confinement time at the maximum depend on the plasma elongation. This effect is illustrated in Fig. 22, which shows the dependence of $\tau$ on $n$ and K. for an elliptical plasma. The maximum confinement increases by a factor of 1.75 for $k=1.5$ and a factor of 4.02 for $k=3$. The corresponding relative densities for maximum confinement are 1.27 and 2.24 respectively. 


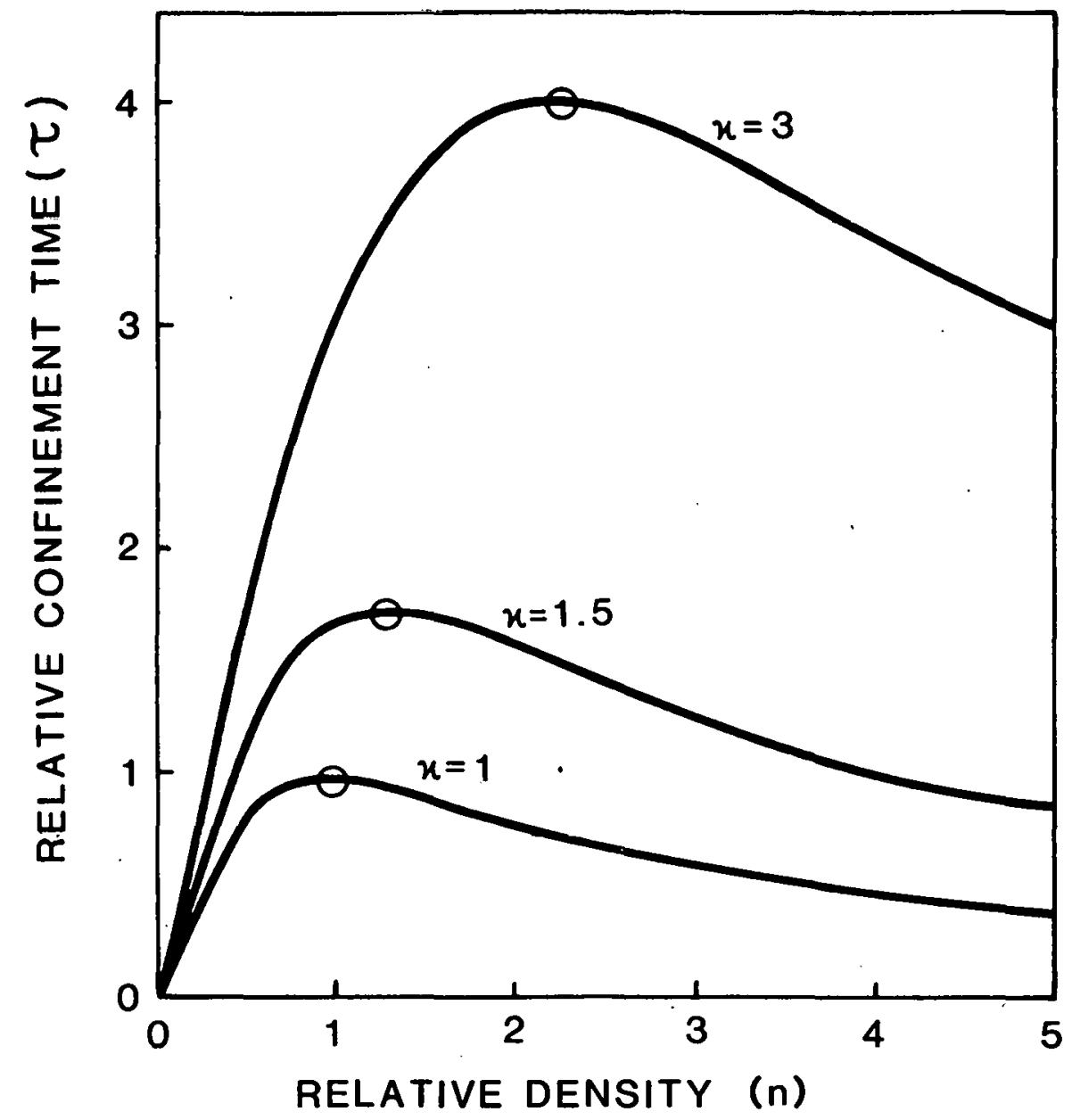

Fig. 22. Effect of neoclassical transport and elongation on energy confinement 